\title{
UNIFORM ALMOST SUB-GAUSSIAN ESTIMATES FOR LINEAR FUNCTIONALS ON CONVEX SETS
}

\author{
B. KLARTAG
}

In honor of Professor V. A. Zalgaller

\begin{abstract}
A well-known consequence of the Brunn-Minkowski inequality says that the distribution of a linear functional on a convex set has a uniformly subexponential tail. That is, for any dimension $n$, any convex set $K \subset \mathbb{R}^{n}$ of volume one, and any linear functional $\varphi: \mathbb{R}^{n} \rightarrow \mathbb{R}$, we have
\end{abstract}

$$
\operatorname{Vol}_{n}\left(\left\{x \in K ;|\varphi(x)|>t\|\varphi\|_{L_{1}(K)}\right\}\right) \leq e^{-c t} \text { for all } t>1,
$$

where $\|\varphi\|_{L_{1}(K)}=\int_{K}|\varphi(x)| d x$ and $c>0$ is a universal constant. In this paper, it is proved that for any dimension $n$ and a convex set $K \subset \mathbb{R}^{n}$ of volume one, there exists a nonzero linear functional $\varphi: \mathbb{R}^{n} \rightarrow \mathbb{R}$ such that

$$
\operatorname{Vol}_{n}\left(\left\{x \in K ;|\varphi(x)|>t\|\varphi\|_{L_{1}(K)}\right\}\right) \leq e^{-c \frac{t^{2}}{\log ^{5}(t+1)}} \text { for all } t>1,
$$

where $c>0$ is a universal constant.

\section{§1. INTRODUCTION}

For two subsets $A, B \subset \mathbb{R}^{n}$ and $\lambda>0$, we denote $\lambda A=\{\lambda x ; x \in A\}$ and $A+B=$ $\{x+y ; x \in A, y \in B\}$. The latter operation is the well-known Minkowski sum of sets. The classical Brunn-Minkowski inequality (see, e.g., [35]) states that for any dimension $n$, any Borel sets $A, B \subset \mathbb{R}^{n}$, and any $0<\lambda<1$, we have

$$
\operatorname{Vol}_{n}(\lambda A+(1-\lambda) B) \geq \operatorname{Vol}_{n}(A)^{\lambda} \operatorname{Vol}_{n}(B)^{1-\lambda},
$$

where $\operatorname{Vol}_{n}$ is the standard Lebesgue measure on $\mathbb{R}^{n}$. A convex body is a compact, convex set with a nonempty interior. Suppose that $K \subset \mathbb{R}^{n}$ is a convex body of volume one, and let $\varphi: \mathbb{R}^{n} \rightarrow \mathbb{R}$ be a nonzero linear functional. Let $M>0$ be such that the set $T=\{x \in$ $\left.\mathbb{R}^{n} ;|\varphi(x)| \leq M\right\}$ satisfies $\operatorname{Vol}_{n}(K \cap T)=\frac{2}{3}$. Clearly $M \leq 3\|\varphi\|_{L_{1}(K)}=3 \int_{K}|\varphi(x)| d x$. An elegant argument by Borell [6] states that the Brunn-Minkowski inequality (1), together with the easily verified inclusion

implies that

$$
\frac{2}{t+1}(K \backslash t T)+\frac{t-1}{t+1}(K \cap T) \subset K \backslash T \text { for all } t \geq 1
$$

$$
\operatorname{Vol}_{n}(\{x \in K ;|\varphi(x)|>t M\}) \leq \frac{1}{3} \frac{1}{2^{\frac{t-1}{2}}} \text { for all } t \geq 1 .
$$

Inequality (2) is a dimension-free subexponential estimate for the tail of the distribution of an arbitrary linear functional on an arbitrary convex body. Recall the definition of the Orlicz norm. Let $\psi:[0, \infty) \rightarrow[0, \infty)$ be a convex, monotone nondecreasing function that

2000 Mathematics Subject Classification. Primary 53A20, 52A21.

Key words and phrases. Brunn-Minkowski inequality, convex body, logarithmic Laplace transform. The author is a Clay Research Fellow and was also supported by NSF (grant no. DMS-0456590). 


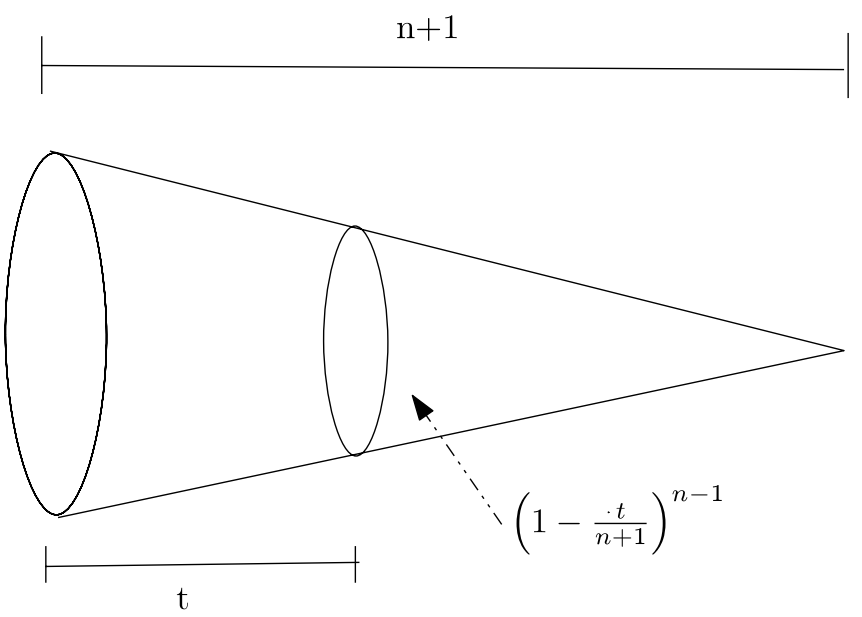

FiguRE 1

vanishes at the origin, and let $(\Omega, \mu)$ be a probability space. For a measurable function $g: \Omega \rightarrow \mathbb{R}$, we denote

$$
\|g\|_{L_{\psi}(\mu)}=\inf \left\{\lambda>0 ; \int_{\Omega} \psi\left(\frac{|g(x)|}{\lambda}\right) d \mu(x) \leq 1\right\} .
$$

For a convex set $K \subset \mathbb{R}^{n}$ of volume one, we write $\|\cdot\|_{L_{\psi}(K)}$ to denote $\|\cdot\|_{L_{\psi}\left(\lambda_{K}\right)}$, where $\lambda_{K}$ is the restriction of the Lebesgue measure on $\mathbb{R}^{n}$ to $K$. We shall mainly consider the Young functions $\psi_{1}(t)=e^{t}-1, \psi_{2}(t)=e^{t^{2}}-1$ and their variants. Inequality (2) now translates as follows: for a convex body $K \subset \mathbb{R}^{n}$ of volume one and a linear functional $\varphi: \mathbb{R}^{n} \rightarrow \mathbb{R}$

$$
\|\varphi\|_{L_{\psi_{1}}(K)} \leq C\|\varphi\|_{L_{1}(K)}
$$

where $C>0$ is some universal constant. We would like to emphasize that the constant $C$ in (3) is a universal constant, independent of $\varphi, K$, and the dimension $n$.

Let us consider an example. Suppose $K \subset \mathbb{R}^{n}$ is a simplex of volume one, with the origin lying in one of its facets. Let $\varphi: \mathbb{R}^{n} \rightarrow \mathbb{R}$ be a nonzero linear functional that is nonnegative on $K$ and vanishes on the facet of $K$ that contains the origin. We assume that $\varphi$ is normalized so that $\|\varphi\|_{L_{1}(K)}=1$. Suppose that $X$ is a random vector that is distributed uniformly over $K$. Then the random variable $\varphi(X)$ has a density that is proportional to the function

$$
t \mapsto \begin{cases}\left(1-\frac{t}{n+1}\right)^{n-1} & \text { if } 0 \leq t \leq n+1, \\ 0 & \text { otherwise. }\end{cases}
$$

Since $(1-t /(n+1))^{n-1} \approx e^{-t}$ for large $n$, the distribution of the random variable $\varphi(X)$ is very close to being an exact exponential when the dimension $n$ is large. Note that there is nothing special about the simplex; any cone over a convex base exhibits such approximately-exponential behavior (see Figure 1).

We conclude that (3) is sharp, in the following strong sense: suppose that $\psi:[0, \infty) \rightarrow$ $[0, \infty)$ is a convex, nondecreasing function that vanishes at the origin. Suppose also that $C>0$ has the property that for any dimension $n$, any convex body $K \subset \mathbb{R}^{n}$ of volume one, and any linear functional $\varphi: \mathbb{R}^{n} \rightarrow \mathbb{R}$, we have $\|\varphi\|_{L_{\psi}(K)} \leq C\|\varphi\|_{L_{1}(K)}$. Then, necessarily, there exist $C^{\prime}, C^{\prime \prime}>0$ such that $\psi(t) \leq C^{\prime} \psi_{1}\left(C^{\prime \prime} t\right)$ for all $t \geq 0$. 
Estimate (3) is the best possible general estimate. However, for some specific classes of convex bodies, there exist much better estimates than (3). Consider, for instance, an ellipsoid $\mathcal{E} \subset \mathbb{R}^{n}$ of volume one, centered at the origin. Let $\varphi: \mathbb{R}^{n} \rightarrow \mathbb{R}$ be a nonzero linear functional. Note that the random variable $\varphi(X)$, where $X$ is distributed uniformly over $\mathcal{E}$, has a density proportional to

$$
t \mapsto\left(1-\frac{a t^{2}}{n}\right)_{+}^{\frac{n-1}{2}} \approx \exp \left(-\frac{a t^{2}}{2}\right),
$$

for some $a>0$. This distribution is very close to the Gaussian distribution. We thus conclude that, for any linear functional $\varphi: \mathbb{R}^{n} \rightarrow \mathbb{R}$,

$$
\|\varphi\|_{L_{\psi_{2}}(\mathcal{E})} \leq C\|\varphi\|_{L_{1}(\mathcal{E})}
$$

where $C>0$ is a universal constant. All nonzero linear functionals are distributed approximately in accordance with the Gaussian law, and hence, for each nonzero linear functional $\varphi$, estimate (4) is essentially sharp. The example of the ellipsoid shows that for some convex bodies, a nonzero linear functional cannot satisfy a dimension-free estimate stronger than $\psi_{2}$. Our purpose in this paper is to prove the following theorem.

Theorem 1.1. Let $n \geq 1$ be an integer, and let $K \subset \mathbb{R}^{n}$ be a convex body of volume one. Then there exists a nonzero linear functional $\varphi: \mathbb{R}^{n} \rightarrow \mathbb{R}$ such that for any $t \geq 1$ we have

$$
\operatorname{Vol}_{n}\left(\left\{x \in K ;|\varphi(x)|>t\|\varphi\|_{L_{1}(K)}\right\}\right)<e^{-c \frac{t^{2}}{\log ^{5}(t+1)}},
$$

where $c>0$ is a universal constant. Equivalently, denote $\psi_{2^{-}}(t)=\exp \left(\frac{t^{2}}{\log ^{5}(t+5)}\right)-1$. Then the linear functional $\varphi$ satisfies

$$
\|\varphi\|_{L_{\psi_{2}}(K)} \leq C\|\varphi\|_{L_{1}(K)},
$$

where $C>0$ is a universal constant.

Therefore, a convex body cannot display "cone-type" behavior in all directions: there always exists a direction in which better, almost sub-Gaussian behavior is observed. Theorem 1.1 is stated only for convex bodies of volume one, with the generalization to general convex bodies being straightforward. An estimate that is slightly weaker than (5) actually holds for "most" linear functionals on $\mathbb{R}^{n}$ in some sense (see precise formulation in Corollary 5.3 below).

Aside from the logarithmic factors, Theorem 1.1 is sharp, as shown by the above discussion. Up to the logarithmic factors, Theorem 1.1 provides an affirmative answer to the following question, attributed to V. Milman in [2] (see also [28, 29]).

Question 1.2 (V. Milman). Does there exist a constant $C>0$ for which the following is true: for any integer $n \geq 1$ and any convex body $K \subset \mathbb{R}^{n}$ of volume one, there exists a nonzero linear functional $\varphi: \mathbb{R}^{n} \rightarrow \mathbb{R}$ such that $\|\varphi\|_{L_{\psi_{2}}(K)} \leq C\|\varphi\|_{L_{1}(K)}$ ?

We briefly review the literature related to Question 1.2. In high-dimensional convex geometry, the significance of dimension-free $\psi_{2}$-estimates is already apparent in Bourgain's bound for the isotropic constant of general convex bodies [8, 9]. In particular, in [9] the isotropic constant of a convex body $K \subset \mathbb{R}^{n}$ was shown to be bounded by a function solely of

$$
\sup _{\varphi \neq 0} \frac{\|\varphi\|_{L_{\psi_{2}}(K)}}{\|\varphi\|_{L_{1}(K)}}
$$

where the supremum runs over all nonzero linear functionals $\varphi: \mathbb{R}^{n} \rightarrow \mathbb{R}$.

An affirmative answer to Question 1.2 has been obtained for some particular classes of convex bodies. Bobkov and Nazarov $[4,5]$ provided a positive answer to Question 1.2 
in the case where $K$ is assumed to be an unconditional convex body. Paouris [28, 29] gave an affirmative answer to Question 1.2 in the case where $K$ is a zonoid, or when $K$ is a convex body with a "small diameter". See [2] for more information on the case of $K$ being the unit ball of $l_{p}^{n}$, for $2 \leq p \leq \infty$.

In the context of arbitrary convex bodies, no general, dimension-free estimates have been obtained, beyond the $\psi_{1}$-estimate (3). Note that it is easy to establish some dimension-dependent estimates for the tail distribution of linear functionals on convex bodies. For instance, from the Brunn-Minkowski inequality it follows that $\|\varphi\|_{L_{\infty}(K)} \leq$ $C n\|\varphi\|_{L_{1}(K)}$ for any $n$-dimensional convex body of volume one and any linear functional $\varphi: \mathbb{R}^{n} \rightarrow \mathbb{R}$, where $C>0$ is a universal constant.

Remarkable progress pertaining to the understanding of mass distributions in highdimensional convex bodies was recently obtained by Paouris [30]. Among the consequences of Paouris' theorem [30], there is a version of Theorem 1.1, without the logarithmic factors, but with $t$ restricted to the range $\left[1, n^{1 / 4}\right]$. This version follows immediately by combining Paouris' theorem [30] with the methods of [29]. Some of our techniques here are related to and influenced by the approach taken by Paouris.

Theorem 1.1 allows generalizations slightly beyond the context of uniform measures on convex sets. Recall that a function $f: \mathbb{R}^{n} \rightarrow[0, \infty)$ is a logarithmically concave function, log-concave in short, if

$$
f(\lambda x+(1-\lambda) y) \geq f(x)^{\lambda} f(y)^{1-\lambda} \text { for all } x, y \in \mathbb{R}^{n}, 0<\lambda<1 .
$$

A basic subclass of log-concave functions consists of the $s$-concave functions for $s>0$. A function $f: \mathbb{R}^{n} \rightarrow[0, \infty)$ is $s$-concave, for some $s>0$, if

$$
f^{\frac{1}{s}}(\lambda x+(1-\lambda) y) \geq \lambda f^{\frac{1}{s}}(x)+(1-\lambda) f^{\frac{1}{s}}(y)
$$

for all $0<\lambda<1$ and for all $x, y \in \mathbb{R}^{n}$ with $f(x), f(y)>0$. The characteristic function of a convex set is an $s$-concave function, for any $s>0$. Theorem 1.1 is not true if we replace uniform measures on convex sets with arbitrary log-concave densities (consider, e.g., the one-dimensional log-concave density $\left.t \mapsto e^{-|t|}\right)$. However, for functions that are $s$-concave the following theorem holds.

Theorem 1.3. Let $\alpha>0$, let $n \geq 1$ be an integer, and let $f: \mathbb{R}^{n} \rightarrow[0, \infty)$ be an $\alpha n$-concave function with $\int f=1$. Denote by $\mu$ the measure whose density is $f$. Then there exists a nonzero linear functional $\varphi: \mathbb{R}^{n} \rightarrow \mathbb{R}$ such that

$$
\mu\left(\left\{x \in \mathbb{R}^{n} ;|\varphi(x)|>t\|\varphi\|_{L_{1}(\mu)}\right\}\right)<e^{-c_{\alpha} \frac{t^{2}}{\log ^{5}(t+1)}}
$$

for any $t \geq 1$, where $c_{\alpha}>0$ is a constant depending solely on $\alpha$.

Our main technical tool here is the logarithmic Laplace transform, as in our previous work [20]. The logarithmic Laplace transform is introduced and discussed in $\S 2$, and then applied in $\S 3$ to the study of log-concave functions with a bounded isotropic constant. $\S 3$ contains the main technical steps of the proof. Theorem 1.1 and Theorem 1.3 are proved in $\S 4$. In $\S 5$ we address questions regarding the behavior of a "typical" linear functional. Throughout this text, unless mentioned otherwise, we use the symbols $c, c^{\prime}, \tilde{c}, \hat{c}, c_{1}, c_{2}, C, C^{\prime}, \tilde{C}, \hat{C}$, etc. to denote various positive universal constants, whose value is not necessarily the same in different appearances.

Acknowledgement. I would like to thank Charles Fefferman for his kind encouragement and for many interesting discussions. I would also like to thank Jean Bourgain and Gady Kozma for valuable discussions on possible applications of the results in this paper. 


\section{$\S 2$. Logarithmic Laplace transform}

In this section we develop a number of estimates related to the logarithmic Laplace transform of $\log$-concave functions. We denote by $|\cdot|$ and $\langle\cdot, \cdot\rangle$ the standard Euclidean norm and scalar product in $\mathbb{R}^{n}$, respectively. We also write $D^{n}=\left\{x \in \mathbb{R}^{n} ;|x| \leq 1\right\}$ and $S^{n-1}=\left\{x \in \mathbb{R}^{n} ;|x|=1\right\}$, for the unit Euclidean ball and the unit sphere in $\mathbb{R}^{n}$, respectively. When the dimension is clear from the context, we may write $D$ in place of $D^{n}$. Let us begin with a very standard lemma.

Lemma 2.1. Let $n \geq 1$ and let $f: \mathbb{R}^{n} \rightarrow[0, \infty)$ be a log-concave function with $0<$ $\int f<\infty$. Then there exist $A, B>0$ such that, for all $x \in \mathbb{R}^{n}$,

$$
f(x) \leq A e^{-B|x|} .
$$

Proof. Since $\int f>0$, there exists $0<\varepsilon<1$ such that $K=\left\{x \in \mathbb{R}^{n} ; f(x)>\varepsilon\right\}$ satisfies $\operatorname{Vol}_{n}(K)>0$. The set $K$ is convex because $f$ is log-concave. Therefore, $K$ has a nonempty interior, since it is a convex set of positive volume. By translating $f$, we may assume that $r D^{n} \subset K$ for some $r>0$. Denote $T=\left\{x \in \mathbb{R}^{n} ; f(x)>\frac{\varepsilon}{2}\right\}$. The set $T$ is convex, $\operatorname{Vol}_{n}(T) \geq \operatorname{Vol}_{n}(K)>0$, and also $\operatorname{Vol}_{n}(T)<\infty$ since $\int f<\infty$. Consequently, $T$ is bounded, being a convex set of finite, positive volume. Thus, for some $R>0$ we have $T \subset \frac{R}{2} D^{n}$. Pick $x \notin R D^{n}$. Then

$$
R \frac{x}{|x|}=\frac{|x|-R}{|x|-r} \cdot r \frac{x}{|x|}+\frac{R-r}{|x|-r} \cdot x
$$

is a convex combination. Note that $R \frac{x}{|x|} \notin T$ and $r \frac{x}{|x|} \in K$. Using (6) and the logconcavity of $f$, we deduce that

$$
\frac{\varepsilon}{2} \geq f\left(R \frac{x}{|x|}\right) \geq f\left(r \frac{x}{|x|}\right)^{\frac{|x|-R}{|x|-r}} \cdot f(x)^{\frac{R-r}{|x|-r}} \geq \varepsilon^{\frac{|x|-R}{|x|-r}} f(x)^{\frac{R-r}{|x|-r}}
$$

In particular,

$$
f(x) \leq \varepsilon\left(\frac{1}{2}\right)^{\frac{|x|-r}{R-r}} \leq A e^{-B|x|} \text { for any } x \notin R D^{n},
$$

with some $A, B>0$ depending on $f$. Note also that $f$ is bounded in $R D^{n}$; otherwise the log-concavity implies that $f=\infty$ on $K$, in contradiction to our assumption that $\int f<\infty$. We conclude that an estimate of the form $f(x) \leq A^{\prime} e^{-B^{\prime}|x|}$, for some numbers $A^{\prime}, B^{\prime}>0$, is true in the entire $\mathbb{R}^{n}$.

For a function $F: \mathbb{R}^{n} \rightarrow \mathbb{R} \cup\{\infty\}$, we write $\operatorname{Dom}(F)=\left\{x \in \mathbb{R}^{n} ; F(x)<\infty\right\}$. Let $f: \mathbb{R}^{n} \rightarrow[0, \infty)$ be a log-concave function with $0<\int f<\infty$. We define $\Upsilon f: \mathbb{R}^{n} \rightarrow$ $\mathbb{R} \cup\{\infty\}$ by setting

$$
\Upsilon f(x)=\log \int_{\mathbb{R}^{n}} e^{\langle x, y\rangle} f(y) \frac{d y}{\int f} .
$$

In this paper "log" stands for the natural logarithm. By Lemma 2.1, the function $\Upsilon f$ is finite in some open neighborhood of the origin. Furthermore, if $x \in \operatorname{Dom}(\Upsilon f)$, then $\tilde{f}(y)=e^{\langle x, y\rangle} f(y)$ is a log-concave function that has a positive, finite integral. Lemma 2.1 implies that $\tilde{f}$ decays exponentially, and hence we may differentiate under the integral sign any finite number of times. We conclude that $\operatorname{Dom}(\Upsilon f)$ is open, and that $\Upsilon f$ is $C^{\infty}$-smooth in $\operatorname{Dom}(\Upsilon f)$.

A function $F: \mathbb{R}^{n} \rightarrow \mathbb{R} \cup\{\infty\}$ is strictly convex if for any $x_{1}, x_{2} \in \operatorname{Dom}(F)$ with $x_{1} \neq x_{2}$, we have

$$
F\left(\frac{x_{1}+x_{2}}{2}\right)<\frac{F\left(x_{1}\right)+F\left(x_{2}\right)}{2} .
$$


The function $\Upsilon f$ as defined in (7) is strictly convex. Indeed, for any two distinct points $x_{1}, x_{2} \in \operatorname{Dom}(\Upsilon f)$,

$$
\int_{\mathbb{R}^{n}} e^{\left\langle\frac{x_{1}+x_{2}}{2}, y\right\rangle} f(y) \frac{d y}{\int f}<\sqrt{\int_{\mathbb{R}^{n}} e^{\left\langle x_{1}, y\right\rangle} f(y) \frac{d y}{\int f} \cdot \int_{\mathbb{R}^{n}} e^{\left\langle x_{2}, y\right\rangle} f(y) \frac{d y}{\int f}}
$$

by the Cauchy-Schwartz inequality. The following lemma is standard. Its straightforward proof can be found, e.g., in [20, Lemma 3.1].

Lemma 2.2. Let $n \geq 1$, and let $f: \mathbb{R}^{n} \rightarrow[0, \infty)$ be a log-concave function with $0<$ $\int f<\infty$. Then, for any $x \in \operatorname{Dom}(\Upsilon f)$, we have

$$
\nabla(\Upsilon f)(x)=\int_{\mathbb{R}^{n}} y d \mu_{x}(y)
$$

where $\mu_{x}$ is the probability measure on $\mathbb{R}^{n}$ whose density is proportional to $y \mapsto e^{\langle x, y\rangle} f(y)$. Additionally,

$$
\operatorname{Hess}(\Upsilon f)(x)=\operatorname{Cov}\left(\mu_{x}\right)=\int_{\mathbb{R}^{n}} y \otimes y d \mu_{x}(y)-\left[\int_{\mathbb{R}^{n}} y d \mu_{x}(y)\right] \otimes\left[\int_{\mathbb{R}^{n}} y d \mu_{x}(y)\right],
$$

the covariance matrix of $\mu_{x}$. Here "Hess" stands for Hessian, and $x \otimes x$ stands for the matrix whose entries are $\left(x_{i} x_{j}\right)_{i, j=1, \ldots, n}$.

A set $K \subset \mathbb{R}^{n}$ is centrally symmetric when $K=-K$. Recall that, for a centrally symmetric convex body $K \subset \mathbb{R}^{n}$, the set

$$
K^{\circ}=\left\{x \in \mathbb{R}^{n} ;\langle x, y\rangle \leq 1 \text { for all } y \in K\right\}
$$

is the polar body of $K$. For a convex body $K \subset \mathbb{R}^{n}$, we denote

$$
\text { v. } \operatorname{rad} .(K)=\left(\frac{\operatorname{Vol}_{n}(K)}{\operatorname{Vol}_{n}\left(D^{n}\right)}\right)^{\frac{1}{n}}
$$

the radius of the Euclidean ball that has the same volume as $K$. Recall that $c_{1}<$ $\sqrt{n} \operatorname{Vol}_{n}\left(D^{n}\right)^{\frac{1}{n}}<c_{2}$ for some universal constants $c_{1}, c_{2}>0$ (see, e.g., the first pages of [31]). Therefore, v. $\operatorname{rad} .(K)$ has the order of magnitude of $\sqrt{n} \operatorname{Vol}_{n}(K)^{1 / n}$.

The following lemma may be interesting in its own right. It is related to the use of $\nabla(\Upsilon f)$ as a "transportation map", an idea developed already in [20]. It may be beneficial, when reading this lemma, to have in mind the simplest example of the function $F(x)=A|x|^{2} / 2$.

Lemma 2.3. Let $n \geq 1$, and let $F: \mathbb{R}^{n} \rightarrow \mathbb{R} \cup\{\infty\}$ be a $C^{2}$-smooth, strictly convex, even function with $F(0)=0$. Fix $t>0$ and set $K=\left\{x \in \mathbb{R}^{n} ; F(x) \leq t\right\}$. Let $A>0,0<\alpha \leq 1 / 2$ and suppose that

$$
\operatorname{det} \operatorname{Hess}(F)(x) \geq A^{n} \text { for } x \in \alpha K \text {. }
$$

Then

$$
\text { v. } \operatorname{rad} .(K) \leq \sqrt{\frac{2}{\alpha A}} \cdot \sqrt{t} .
$$

Proof. Fix $x \in \alpha K$. Since $F$ is convex, the graph of $F$ lies above the supporting hyperplane to $F$ at $x$. In particular,

$$
F(x)+\langle\nabla F(x), 2 x-x\rangle \leq F(2 x) \leq t,
$$

because $2 x \in 2 \alpha K \subset K$. Note that $F$ is nonnegative, being a convex, even function that vanishes at the origin. In particular, we have $F(x) \geq 0$, and (9) implies that

$$
\langle\nabla F(x), x\rangle \leq t \text {. }
$$


Next, fix $y \in K$. Using (10) and the convexity of $F$, we obtain

$$
t \geq F(y) \geq F(x)+\langle\nabla F(x), y-x\rangle \geq F(x)+\langle\nabla F(x), y\rangle-t .
$$

Since $F(x) \geq 0$, we deduce from (11) that

$$
\langle\nabla F(x), y\rangle \leq 2 t \text { for all } x \in \alpha K, y \in K .
$$

Denote $K^{\prime}=\{\nabla F(x) ; x \in \alpha K\}$. Then (12) is interpreted as

$$
K^{\prime} \subset 2 t K^{\circ} \text {. }
$$

Since the function $F$ is strictly convex on $K$, its gradient is a one-to-one map. By substituting $x=\nabla F(y)$, we obtain

$$
\operatorname{Vol}_{n}\left(K^{\prime}\right)=\int_{\{\nabla F(y) ; y \in \alpha K\}} d x=\int_{\alpha K} \operatorname{det} \operatorname{Hess}(F)(y) d y \geq \operatorname{Vol}_{n}(\alpha K) A^{n},
$$

where we have used (8). By combining (13) and (14) we conclude that

$$
\operatorname{Vol}_{n}\left(K^{\circ}\right) \geq\left(\frac{\alpha A}{2 t}\right)^{n} \operatorname{Vol}_{n}(K) \text {. }
$$

The set $K$ is centrally symmetric and convex. By the Santaló inequality (see [34] or $[1,24])$, we know that v.rad. $(K) \mathrm{v} \cdot \operatorname{rad} .\left(K^{\circ}\right) \leq 1$. Thus, $(15)$ implies that

$$
\text { v. } \operatorname{rad} .(K) \leq \frac{\sqrt{2 t}}{\sqrt{\alpha A}} .
$$

The lemma is proved.

Next, we review some of the basic, nontrivial properties of log-concave functions. Suppose $f: \mathbb{R}^{n} \rightarrow[0, \infty)$ is a log-concave function with $0<\int f<\infty$. By the PrékopaLeindler inequality (see $[32,22,33]$ or, e.g., the first pages of [31]),

$$
\sqrt{\int_{\lambda A+(1-\lambda) B} f(x) d x} \geq\left(\int_{A} f(x) d x\right)^{\lambda}\left(\int_{B} f(x) d x\right)^{1-\lambda}
$$

for any nonempty Borel sets $A, B \subset \mathbb{R}^{n}$ and $0<\lambda<1$. Denote by $\mu$ the measure whose density is $f$, and let $\varphi: \mathbb{R}^{n} \rightarrow \mathbb{R}$ be a linear functional. As was explained in $\S 1$ for the case of convex bodies, Borell's lemma [6] states that

$$
\|\varphi\|_{L_{\psi_{1}}(\mu)} \leq C\|\varphi\|_{L_{1}(\mu)}
$$

where $C>0$ is a universal constant. We conclude from Borell's lemma, in particular, that $\|\varphi\|_{L_{2}(\mu)} \leq C\|\varphi\|_{L_{1}(\mu)}$. Furthermore, let $M>0$ be such that

$$
\mu\left(\left\{x \in \mathbb{R}^{n} ;|\varphi(x)| \leq M\right\}\right)=\frac{2}{3} .
$$

Then $c\|\varphi\|_{L_{1}(\mu)} \leq M \leq 3\|\varphi\|_{L_{1}(\mu)}$. Another consequence of (16) is that the marginal function $\left(x_{1}, \ldots, x_{k}\right) \mapsto \int f\left(x_{1}, \ldots, x_{n}\right) d x_{k+1} \ldots d x_{n}$ is log-concave. Consequently, the convolution of two log-concave functions is again a log-concave function, a fact that was already known to Lekkerkerker [23] in one dimension, and to Davidovich, Korenblyum, and Khatset [12] in the general case. Next, suppose that $f: \mathbb{R}^{n} \rightarrow[0, \infty)$ is not only $\log$-concave, but also $s$-concave for some $s>0$. Then (16) may be strengthened as follows:

$$
\left(\int_{\alpha A+\beta B} f(x) d x\right)^{\frac{1}{n+s}} \geq \alpha\left(\int_{A} f(x) d x\right)^{\frac{1}{n+s}}+\beta\left(\int_{B} f(x) d x\right)^{\frac{1}{n+s}}
$$

for any Borel sets $A, B \subset \mathbb{R}^{n}$ and $\alpha, \beta>0$. Inequality (18) in $n$ dimensions was proved by Dinghas [13, 14], by Borell [7], and by Brascamp and Lieb [11]. It follows directly from the Brunn-Minkowski inequality (1); see [19]. The one-dimensional case of (18) is due 
to Henstock and Macbeath [16]. The same line of reasoning also leads to the conclusion that the convolution of an $s_{1}$-concave function on $\mathbb{R}^{n}$ with an $s_{2}$-concave function on $\mathbb{R}^{n}$ is an $\left(s_{1}+s_{2}+n\right)$-concave function (see, e.g., [7]).

The next lemma is standard, and is contained, e.g., in [17, Lemma 2.3]. Rather than referring the reader to the proof in [17], here we shall present a simpler proof that we learned from Mark Rudelson.

Lemma 2.4. Let $n \geq 1$, let $K \subset \mathbb{R}^{n}$ be a centrally-symmetric convex body, and let $x \in \mathbb{R}^{n}, t>0$. Suppose that $\sup _{y \in K}\langle x, y\rangle \geq t$. Denote

$$
A=\left\{y \in K ;\langle x, y\rangle \geq \frac{t}{2}\right\} \text {. }
$$

Then

$$
\operatorname{Vol}_{n}(A) \geq 2^{-n-1} \operatorname{Vol}_{n}(K) .
$$

Proof. Denote $K^{+}=\{y \in K ;\langle x, y\rangle \geq 0\}$. Let $y_{0} \in K$ be a point such that $\left\langle x, y_{0}\right\rangle \geq t$. Since $y_{0} \in K$ and $K^{+} \subset K$, by convexity,

$$
\frac{1}{2} y_{0}+\frac{1}{2} K^{+} \subset K
$$

For any $y \in\left(y_{0}+K^{+}\right) / 2$ we have $\langle x, y\rangle \geq t / 2$. Therefore, $\left(y_{0}+K^{+}\right) / 2 \subset A$. Hence,

$$
\operatorname{Vol}_{n}(A) \geq \operatorname{Vol}_{n}\left(\frac{y_{0}+K^{+}}{2}\right)=2^{-n} \operatorname{Vol}_{n}\left(K^{+}\right)=2^{-n-1} \operatorname{Vol}_{n}(K) .
$$

Later on, we shall make use of the following standard estimate for the gamma function. Begin with the routinely-verified fact that, for any $n>0, A>1$, and $t>A n$, we have $t^{n} e^{-t} \leq(A n)^{n} e^{-A n} \exp \left(-\left(1-\frac{1}{A}\right)(t-A n)\right)$. Consequently,

$$
\int_{A n}^{\infty} t^{n} e^{-t} d t \leq(A n)^{n} e^{-A n} \frac{A}{A-1} \text { for any } A>1
$$

Our next lemma is an application of Laplace's asymptotic method and is a direct extension of [21, Lemma 2.1].

Lemma 2.5. Let $g:[0, \infty) \rightarrow[0, \infty]$ be a monotone nondecreasing, continuous, convex function such that $g(0)=0$ and $g \not \equiv 0$. Suppose that $g$ is continuously differentiable on $\operatorname{Dom}(g)$. Let $n \geq 1$ be an integer, and let $t_{0}>0$ be a point such that

$$
e^{-g\left(t_{0}\right)} t_{0}^{n}=\sup _{t>0} e^{-g(t)} t^{n}
$$

Then $t_{0}$ exists and is unique. Furthermore, $t_{0}$ satisfies

(i) $g\left(t_{0}\right) \leq n$;

(ii) $\int_{5 t_{0}}^{\infty} e^{-g(t)} t^{n} d t \leq e^{-n} \int_{0}^{\infty} e^{-g(t)} t^{n} d t$;

(iii) if $a \in \mathbb{R}$ is such that $\left|a t_{0}\right| \leq \frac{n}{20}$, then

$$
\int_{0}^{\infty} e^{a t-g(t)} t^{n} d t \leq e^{n} \int_{0}^{\infty} e^{-g(t)} t^{n} d t .
$$

Proof. The function $g$ is a nonconstant, convex function. Hence, $g(t)$ tends to infinity at least linearly fast as $t \rightarrow \infty$. Consequently, the function $\varphi(t)=-g(t)+n \log t$ tends to $-\infty$ as $t \rightarrow 0$ or $t \rightarrow \infty$. Since $\varphi$ is continuous, its finite maximum is attained. Furthermore, since $\varphi$ is strictly concave, the maximum is attained at a unique point $t_{0} \in \operatorname{Dom}(g)$. Thus, a point $t_{0}>0$ that satisfies (20) exists and is unique. Since $\varphi^{\prime}\left(t_{0}\right)=0$, we have

$$
g^{\prime}\left(t_{0}\right) t_{0}=n
$$


By convexity, $g^{\prime}$ is monotone nondecreasing, whence $g^{\prime}(t) \leq \frac{n}{t_{0}}$ for any $t<t_{0}$. Therefore,

$$
g\left(t_{0}\right)=g(0)+\int_{0}^{t_{0}} g^{\prime}(t) d t \leq \int_{0}^{t_{0}} \frac{n}{t_{0}} d t=n .
$$

This proves (i). Next, we prove (ii). Since $g$ is monotone nondecreasing, we have

$$
\int_{0}^{\infty} e^{-g(t)} t^{n} d t \geq e^{-g\left(t_{0}\right)} \int_{0}^{t_{0}} t^{n} d t=\frac{1}{n+1} e^{-g\left(t_{0}\right)} t_{0}^{n+1}
$$

The convexity of $g$ and (21) imply that, for any $t>0$,

$$
g(t) \geq g\left(t_{0}\right)+\frac{n}{t_{0}}\left(t-t_{0}\right)
$$

Therefore,

$$
\int_{5 t_{0}}^{\infty} e^{-g(t)} t^{n} d t \leq e^{n-g\left(t_{0}\right)} \int_{5 t_{0}}^{\infty} e^{-\frac{n}{t_{0}} t} t^{n} d t=\frac{e^{n}}{n^{n+1}} e^{-g\left(t_{0}\right)} t_{0}^{n+1} \int_{5 n}^{\infty} e^{-t} t^{n} d t .
$$

From (24), (22), and the case where $A=5$ in (19), we conclude that

$$
\int_{5 t_{0}}^{\infty} e^{-g(t)} t^{n} d t \leq \frac{5}{4} \frac{e^{n}}{n^{n+1}}\left(\frac{5 n}{e^{5}}\right)^{n} e^{-g\left(t_{0}\right)} t_{0}^{n+1} \leq e^{-n} \int_{0}^{\infty} e^{-g(t)} t^{n} d t
$$

for $n \geq 1$. This completes the proof of (ii). Now we prove (iii). Denote $\psi(t)=g(t)-a t$; this is a convex function. By (21), and since $a t_{0} \leq \frac{n}{20}$, we know that

$$
\psi^{\prime}\left(t_{0}\right) \geq \frac{19 n}{20 t_{0}} .
$$

The convexity of $\psi$ implies that $\psi(t) \geq \psi\left(t_{0}\right)+\frac{19 n}{20 t_{0}}\left(t-t_{0}\right)$ for $t>t_{0}$. Thus,

$$
\begin{aligned}
\int_{5 t_{0}}^{\infty} e^{-\psi(t)} t^{n} d t & \leq e^{\frac{19 n}{20}-\psi\left(t_{0}\right)} \int_{5 t_{0}}^{\infty} e^{-\frac{19 n}{20 t_{0}} t} t^{n} d t \leq e^{-g\left(t_{0}\right)} e^{\frac{19 n}{20}+a t_{0}}\left(\frac{20 t_{0}}{19 n}\right)^{n+1} \int_{4 n}^{\infty} e^{-t} t^{n} d t \\
& \leq \frac{t_{0}^{n+1} e^{-g\left(t_{0}\right)}}{n} \frac{e^{n}}{n^{n}}\left(\frac{20}{19}\right)^{n+1} \cdot \frac{4}{3}\left(\frac{4 n}{e^{4}}\right)^{n} \\
& \leq \frac{t_{0}^{n+1} e^{-g\left(t_{0}\right)}}{n+1} \frac{e^{n}}{2} \leq \frac{e^{n}}{2} \int_{0}^{\infty} e^{-g(t)} t^{n} d t
\end{aligned}
$$

for any $n \geq 1$, where we have used (22) as well as (19). Consequently,

$$
\int_{5 t_{0}}^{\infty} e^{a t-g(t)} t^{n} \leq \frac{e^{n}}{2} \int_{0}^{\infty} e^{-g(t)} t^{n} d t
$$

Moreover, since $5\left|a t_{0}\right| \leq \frac{1}{4}$, we have

$$
\int_{0}^{5 t_{0}} e^{a t-g(t)} t^{n} \leq e^{5\left|a t_{0}\right|} \int_{0}^{5 t_{0}} e^{-g(t)} t^{n} d t \leq \frac{e^{n}}{2} \int_{0}^{\infty} e^{-g(t)} t^{n} d t .
$$

By adding (25) to (26), we obtain the third part of the lemma.

Let $f: \mathbb{R}^{n} \rightarrow[0, \infty)$ be an even, log-concave function. We define

$$
K(f)=\left\{x \in \mathbb{R}^{n} ; f(x) \geq e^{-n} f(0)\right\} .
$$

The set $K(f)$ is convex and centrally symmetric, and it has a nonempty interior if $\int f>0$. The next lemma is, again, very similar to the methods in [21].

Lemma 2.6. Let $n \geq 2$ be an integer, and let $f: \mathbb{R}^{n} \rightarrow[0, \infty)$ be an even, continuously differentiable, log-concave function with $0<\int f<\infty$. Then:

(i) $\int_{5 K(f)} f(y) d y \geq\left(1-e^{-(n-1)}\right) \int_{\mathbb{R}^{n}} f(y) d y$; 
(ii) if $x \in \mathbb{R}^{n}$ is such that $\sup _{y \in K(f)}\langle x, y\rangle \leq \frac{n}{20}$, then

$$
\int_{\mathbb{R}^{n}} e^{\langle x, y\rangle} f(y) d y \leq e^{n} \int_{\mathbb{R}^{n}} f(y) d y
$$

Proof. Since $f$ is even and log-concave, and $\int f>0$, we have $f(0)>0$. We divide $f$ by $f(0)$, and assume from now on that $f(0)=1$. For a unit vector $\theta \in S^{n-1}$, define $g_{\theta}(t)=-\log f(t \theta)$. Since $f$ is even, and log-concave, and $f(0)=1$, we conclude that $g_{\theta}:[0, \infty) \rightarrow[0, \infty]$ is monotone nondecreasing and convex, and $g_{\theta}(0)=0$. The function $g_{\theta}$ is also continuous on $[0, \infty)$ and continuously differentiable on Dom $\left(g_{\theta}\right)$. By Lemma $2.1, g_{\theta} \not \equiv 0$. Thus, the function $g_{\theta}$ and the integer $n-1$ satisfy the requirements of Lemma 2.5. Let $t_{0}(\theta)$ be the point $t_{0}$ from Lemma 2.5, corresponding to $g_{\theta}$ and $n-1$. By Lemma 2.5(i), for any $\theta \in S^{n-1}$, we have

$$
g_{\theta}\left(t_{0}(\theta)\right) \leq n-1 \leq n .
$$

Consequently,

$$
t_{0}(\theta) \theta \in K(f) \text { for all } \theta \in S^{n-1} .
$$

For a set $K \subset \mathbb{R}^{n}$, we denote by $1_{K}$ the characteristic function of $K$. We integrate in polar coordinates, and use (27) and Lemma 2.5(ii), to obtain

$$
\begin{aligned}
\int_{5 K(f)} f(y) d y & =\int_{S^{n-1}} \int_{0}^{\infty} 1_{5 K(f)}(t \theta) f(t \theta) t^{n-1} d t d \theta \\
& \geq \int_{S^{n-1}} \int_{0}^{5 t_{0}(\theta)} t^{n-1} e^{-g_{\theta}(t)} d t d \theta \\
& \geq\left(1-e^{-(n-1)}\right) \int_{S^{n-1}} \int_{0}^{\infty} t^{n-1} e^{-g_{\theta}(t)} d t d \theta \\
& =\left(1-e^{-(n-1)}\right) \int_{\mathbb{R}^{n}} f(y) d y .
\end{aligned}
$$

This proves (i). We move to (ii). Our assumptions in (ii) and (27) imply that

$$
\left|\langle x, \theta\rangle t_{0}(\theta)\right| \leq \frac{n}{20} \text { for any } \theta \in S^{n-1}
$$

(we have also used the fact that $K(f)$ is centrally symmetric). Using (28) and Lemma 2.5 (iii), we see that

$$
\int_{0}^{\infty} e^{\langle x, \theta\rangle t} t^{n-1} e^{-g_{\theta}(t)} d t \leq e^{n-1} \int_{0}^{\infty} t^{n-1} e^{-g_{\theta}(t)} d t
$$

for any $\theta \in S^{n-1}$. By integrating the last inequality in polar coordinates, we conclude that

$$
\begin{aligned}
\int_{\mathbb{R}^{n}} e^{\langle x, y\rangle} f(y) d y & =\int_{S^{n-1}} \int_{0}^{\infty} e^{\langle x, t \theta\rangle} f(t \theta) t^{n-1} d t d \theta \\
& =\int_{S^{n-1}} \int_{0}^{\infty} e^{\langle x, \theta\rangle t} t^{n-1} e^{-g_{\theta}(t)} d t d \theta \leq e^{n-1} \int_{S^{n-1}} \int_{0}^{\infty} t^{n-1} e^{-g_{\theta}(t)} d t d \theta \\
& \leq e^{n} \int_{\mathbb{R}^{n}} f(x) d x
\end{aligned}
$$

This establishes (ii). The proof of the lemma is complete. 
For convenience, our notation treats both $f$ and $\Upsilon f$ as functions defined on $\mathbb{R}^{n}$. It is important to keep in mind that, in principle, $\Upsilon f$ is defined on the dual space. In particular, for any linear map $T: \mathbb{R}^{n} \rightarrow \mathbb{R}^{n}$ and a function $f: \mathbb{R}^{n} \rightarrow[0, \infty)$,

$$
\Upsilon(f \circ T)=(\Upsilon f) \circ\left(T^{*}\right)^{-1},
$$

where $(f \circ T)(x)=f(T(x))$. Also, note that $K(f \circ T)=T^{-1} K(f)$. In addition, for any $a>0$ we clearly have

$$
\Upsilon(a f)=\Upsilon f, \quad K(a f)=K(f) .
$$

Lemma 2.7. Let $n \geq 2$ be an integer, and let $f: \mathbb{R}^{n} \rightarrow[0, \infty)$ be an even, continuously differentiable, log-concave function with $0<\int f<\infty$. The following statements are true:

(i) For any $x \in \mathbb{R}^{n}$,

$$
\Upsilon f(x) \leq n \Rightarrow \sup _{y \in K(f)}\langle x, y\rangle \leq C n .
$$

(ii) For any $x \in \mathbb{R}^{n}$,

$$
\sup _{y \in K(f)}\langle x, y\rangle \leq c n \Rightarrow \Upsilon f(x) \leq n .
$$

(iii) For any $x \in \mathbb{R}^{n}$,

$$
\Upsilon f(C x) \leq n \Rightarrow \sup _{y \in \mathbb{R}^{n}} e^{\langle x, y\rangle} f(y) \leq e^{n} f(0) .
$$

Here c, $C>0$ are universal constants.

Proof. By (30), we may multiply $f$ by a positive constant and assume that $f(0)=1$. Furthermore, for $\delta>0$ denote $\tau_{\delta}(x)=\delta x$. By (29) and the discussion around it, we may replace $f$ with $f \circ \tau_{\delta}$ for an appropriate $\delta>0$, and assume that $\int f=1$.

We begin with proving (i). Let $x \in \mathbb{R}^{n}$ be such that

$$
\sup _{y \in K(f)}\langle x, y\rangle \geq C_{1} n
$$

for a universal constant $C_{1}$ to be chosen later. In order to prove (i), it suffices to show that $\Upsilon f(x)>n$. That is, it suffices to prove that

$$
\int_{\mathbb{R}^{n}} e^{\langle x, y\rangle} f(y) d y>e^{n} .
$$

By (31) and Lemma 2.4, the set

$$
A=K(f) \cap\left\{y \in \mathbb{R}^{n} ;\langle x, y\rangle \geq \frac{C_{1}}{2} n\right\}
$$

satisfies $\operatorname{Vol}_{n}(A) \geq 2^{-n-1} \operatorname{Vol}_{n}(K(f))$. The function $f$ is even and log-concave, and $f(0)=1$. Hence, $f(y)=\sqrt{f(y) f(-y)} \leq f(0)=1$ for all $y \in \mathbb{R}^{n}$, and $\sup f=1$. By Lemma 2.6(i),

$$
5^{n} \operatorname{Vol}_{n}(K(f)) \geq \int_{5 K(f)} f(x) d x \geq 1-e^{-(n-1)} \geq e^{-n} .
$$

We conclude that

$$
\operatorname{Vol}_{n}(A) \geq 2^{-n-1} \operatorname{Vol}_{n}(K(f)) \geq e^{-c^{\prime} n}
$$

Note that

$$
\int_{\mathbb{R}^{n}} e^{\langle x, y\rangle} f(y) d y \geq \int_{A} e^{\langle x, y\rangle} f(y) d y \geq \operatorname{Vol}_{n}(A) \cdot \min _{y \in A} e^{\langle x, y\rangle} \cdot \min _{y \in A} f(y) .
$$


By using (33), (34) and the definition of $K(f)$, we deduce that

$$
\int_{\mathbb{R}^{n}} e^{\langle x, y\rangle} f(y) d y \geq \exp \left[\left(\frac{C_{1}}{2}-c^{\prime}-1\right) n\right]>e^{n}
$$

for a sufficiently large universal constant $C_{1}>0$. Thus, (32) is proved. This completes the proof of (i). To prove (ii), suppose that $x \in \mathbb{R}^{n}$ is such that

$$
\sup _{y \in K(f)}\langle x, y\rangle \leq \frac{n}{20} .
$$

By Lemma 2.6(ii), the inequality (35) entails

$$
\int_{\mathbb{R}^{n}} e^{\langle x, y\rangle} f(y) d y \leq e^{n}
$$

Thus, (35) implies that $\Upsilon f(x) \leq n$, and (ii) is proved. It remains to establish (iii). We shall assume that $x \in \mathbb{R}^{n}$ satisfies

$$
\Upsilon f\left(C_{2} x\right) \leq n
$$

for a sufficiently large constant $C_{2}>0$ to be specified later on. In accordance with part (i) of the present lemma, which has already been proved, we have

$$
\sup _{y \in K(f)}\langle x, y\rangle \leq \frac{C}{C_{2}} n \leq n,
$$

where we assume, as we may, that the constant $C_{2}$ is larger than the constant $C$ occurring in part (i). The function $f$ is continuous. Therefore, whenever $y \in \partial K(f)$, we have $f(y)=e^{-n} f(0)=e^{-n}$. Denote $g(y)=e^{\langle x, y\rangle} f(y)$; this is a continuous log-concave function. From (37) we see that

$$
g(y)=e^{\langle x, y\rangle} f(y)=e^{\langle x, y\rangle} e^{-n} \leq 1
$$

for all $y \in \partial K(f)$. Let $y \notin K(f)$. Then $f(y)<e^{-n}$. Since $f$ is continuous and $f(0)=1$, there exists $0<\theta<1$ such that $f(\theta y)=e^{-n}$. Suppose that $0<\theta<1$ is the maximal possible number such that $f(\theta y)=e^{-n}$. Then

$$
\theta y \in \partial K(f) \text {. }
$$

By (38), we know that $g(\theta y) \leq 1$, and the log-concavity of $g$ implies

$$
1 \geq g(\theta y) \geq g(y)^{\theta} g(0)^{1-\theta}=g(y)^{\theta} .
$$

Consequently, $g(y) \leq 1$ for all $y \notin K(f)$. Since $0 \in K(f)$ and $g(0)=1$,

$$
\sup _{y \in \mathbb{R}^{n}} e^{\langle x, y\rangle} f(y)=\sup _{y \in \mathbb{R}^{n}} g(y)=\sup _{y \in K(f)} g(y) \leq \sup _{y \in K(f)} e^{\langle x, y\rangle} \cdot \sup _{y \in K(f)} f(y) .
$$

By combining this with (37), we conclude that

$$
\sup _{y \in \mathbb{R}^{n}} e^{\langle x, y\rangle} f(y) \leq e^{n} \sup _{y \in K(f)} f(y)=e^{n} .
$$

This completes the proof of (iii). Thus, the lemma is proved.

Lemma 2.8. Let $n \geq 2$, and let $f: \mathbb{R}^{n} \rightarrow[0, \infty)$ be an even, log-concave function with $\int f=1$. Let $K=\left\{x \in \mathbb{R}^{n} ; \Upsilon f(x) \leq n\right\}$. Then

$$
c \sqrt{n} f(0)^{\frac{1}{n}} \leq \mathrm{v} \cdot \operatorname{rad} .(K) \leq C \sqrt{n} f(0)^{\frac{1}{n}},
$$

where $c, C>0$ are universal constants. 
Proof. Suppose first that $f$ is continuously differentiable. By the definition of $K(f)$,

$$
e^{-n} f(0) \cdot \operatorname{Vol}_{n}(K(f)) \leq \int_{K(f)} f(x) d x \leq \int_{\mathbb{R}^{n}} f(x) d x=1
$$

Also, by Lemma 2.6(i),

$$
f(0) \operatorname{Vol}_{n}(5 K(f)) \geq \int_{5 K(f)} f(x) d x \geq\left(1-e^{-(n-1)}\right) \int f \geq e^{-n} .
$$

Since $\operatorname{Vol}_{n}(D)^{-\frac{1}{n}}$ has the order of magnitude of $\sqrt{n}$, from (40) and (41) we deduce that

$$
c \frac{\sqrt{n}}{f(0)^{\frac{1}{n}}}<\text { v. } \operatorname{rad} .(K(f))<C \frac{\sqrt{n}}{f(0)^{\frac{1}{n}}} .
$$

Now, by (i) and (ii) of Lemma 2.7,

$$
\tilde{c} n[K(f)]^{\circ} \subset K \subset \tilde{C} n[K(f)]^{\circ} .
$$

By Santaló's inequality (see [34] or [1, 24]) and Bourgain-Milman's inequality [10], from (43) we conclude that

$$
\hat{c} \frac{n}{\text { v. } \operatorname{rad} .(K(f))} \leq \text { v.rad. }(K) \leq \hat{C} \frac{n}{\text { v. } \operatorname{rad} .(K(f))} .
$$

Comparing (44) with (42), we obtain the desired inequality (39) in the case where $f$ is continuously differentiable. This completes the proof for the case where $f$ is a smooth function.

The general case is settled by using a standard approximation argument. Denote $g_{\varepsilon}(x)=(2 \pi \varepsilon)^{-n / 2} \exp \left(-|x|^{2} / 2 \varepsilon\right)$. Then $g_{\varepsilon}$ is log-concave. Since $f$ is also log-concave, so is the convolution $f_{\varepsilon}=f * g_{\varepsilon}$. The function $f_{\varepsilon}$ is $C^{\infty}$-smooth, even, log-concave, and such that $\int f_{\varepsilon}=1$. Thus, the conclusion of the lemma is already proved for $f_{\varepsilon}$. Since $f_{\varepsilon} \rightarrow f$ in the $w^{*}$-topology as $\varepsilon \rightarrow 0$, the conclusions of the lemma also hold true for $f$. The proof is complete.

\section{§3. BOUndED ISOTROPIC CONSTANT}

Let $f: \mathbb{R}^{n} \rightarrow[0, \infty)$ be a log-concave function with $0<\int f<\infty$. Recall that the covariance matrix of $f$ is the matrix $\operatorname{Cov}(f)=\left(\operatorname{Cov}_{i, j}(f)\right)_{i, j=1, \ldots, n}$ whose entries are

$$
\operatorname{Cov}_{i, j}(f)=\int_{\mathbb{R}^{n}} x_{i} x_{j} f(x) \frac{d x}{\int f}-\int_{\mathbb{R}^{n}} x_{i} f(x) \frac{d x}{\int f} \cdot \int_{\mathbb{R}^{n}} x_{j} f(x) \frac{d x}{\int f},
$$

where, as usual, $x=\left(x_{1}, \ldots, x_{n}\right)$ are coordinates in $\mathbb{R}^{n}$. The covariance matrix is well defined by Lemma 2.1. We say that $f$ is isotropic, or that $f$ is in isotropic position, if the barycenter of $f$ lies at the origin and $\operatorname{Cov}(f)$ is a scalar matrix. Note that, for any logconcave function $f: \mathbb{R}^{n} \rightarrow[0, \infty)$ with $0<\int f<\infty$, there exists a volume-preserving affine transformation $T: \mathbb{R}^{n} \rightarrow \mathbb{R}^{n}$ such that $f \circ T$ is isotropic (see, e.g., [26]). The isotropic constant of $f$ is defined as

$$
L_{f}=\left(\frac{\sup _{x \in \mathbb{R}^{n}} f(x)}{\int_{\mathbb{R}^{n}} f(x) d x}\right)^{\frac{1}{n}}(\operatorname{det} \operatorname{Cov}(f))^{\frac{1}{2 n}} .
$$

We refer the reader, e.g., to [26] for a thorough review of the isotropic position and the isotropic constant of convex bodies. Clearly, $L_{f}=L_{\tilde{f}}$ if $\tilde{f}(x)=f\left(x-x_{0}\right)$ for some $x_{0} \in \mathbb{R}^{n}$, and $L_{f}=L_{a f}$ for any $a>0$. Note also that $L_{f}=L_{f \circ T}$ for any linear transformation $T \in G L_{n}(\mathbb{R})$. The following lemma is well known (it is almost identical, e.g., to [26, Lemma 4.1]). Since our definitions are not entirely standard, we reproduce its short proof below. 
Lemma 3.1. Let $n \geq 1$ be an integer, and let $f: \mathbb{R}^{n} \rightarrow[0, \infty)$ be a log-concave function with $0<\int f<\infty$. Then

where $c>0$ is a universal constant.

$$
L_{f}>c
$$

Proof. By translating $f$, if necessary, we may assume that the barycenter of $f$ is at the origin. We may further replace $f$ with $a f \circ T$, for suitable $a>0$ and $T \in G L_{n}(\mathbb{R})$, and suppose that $\sup f=\int f=1$ and that $f$ is isotropic. Consequently, $\operatorname{Cov}(f)=L_{f}^{2} \operatorname{Id}$ and

$$
n L_{f}^{2}=\int_{\mathbb{R}^{n}}|x|^{2} f(x) d x=\int_{0}^{\infty} \int_{\mathbb{R}^{n} \backslash \sqrt{t} D} f(x) d x d t=\int_{0}^{\infty}\left[1-\int_{\sqrt{t} D} f(x) d x\right] d t,
$$

where we have used the fact that $\int f=1$, as well as the identity $\int g d \mu=\int_{0}^{\infty} \mu\{x ; g(x) \geq$ $t\} d t$, valid for any measure $\mu$ and a nonnegative measurable function $g$. Denote $\kappa_{n}=$ $\frac{\pi^{n / 2}}{\Gamma\left(\frac{n}{2}+1\right)}=\operatorname{Vol}_{n}\left(D^{n}\right)$, and recall that $c<\sqrt{n} \kappa_{n}^{\frac{1}{n}}<C$. Since $\sup f=1$, we have

$$
n L_{f}^{2} \geq \int_{0}^{\infty} \max \left\{1-\operatorname{Vol}_{n}(\sqrt{t} D), 0\right\} d t=\int_{0}^{\kappa_{n}^{-2 / n}}\left(1-t^{n / 2} \kappa_{n}\right) d t=\frac{n}{n+2} \kappa_{n}^{-\frac{2}{n}}>c n .
$$

Thus, $L_{f}>c^{\prime}$, and the proof is complete.

Note that the log-concavity assumption was barely used in the proof of Lemma 3.1. For $0<\varepsilon \leq 1$ and a log-concave function $f: \mathbb{R}^{n} \rightarrow[0, \infty)$ with $0<\int f<1$, we define

$$
\hat{T}_{\varepsilon}(f)=\left\{x \in \mathbb{R}^{n} ; \Upsilon f\left(\frac{2^{i / 2}}{i^{2+\varepsilon}} x\right) \leq 2^{i} \text { for } i=1, \ldots,\left\lfloor\log _{2} n\right\rfloor\right\} .
$$

The set $\hat{T}_{\varepsilon}(f)$ is convex. Our main goal in the next few pages is to show that $\hat{T}_{\varepsilon}(f)$ is sufficiently large. This is the crucial step in the proof of Theorem 1.1. The following lemma serves to motivate the definition of $\hat{T}_{\varepsilon}(f)$ and to demonstrate the usefulness of the desired lower bound for the volume of $\hat{T}_{\varepsilon}(f)$.

Lemma 3.2. Suppose $0<\varepsilon \leq 1, \theta \in \mathbb{R}^{n}$, and $M>0$. Let $n \geq 1$ be an integer, and let $f: \mathbb{R}^{n} \rightarrow[0, \infty)$ be an even, log-concave function with $\int f=1$. Denote by $\mu$ the measure whose density is $f$. Suppose that $\theta / M \in \hat{T}_{\varepsilon}(f)$. Then, for any $1 \leq t \leq \sqrt{n} \log ^{2+\varepsilon / 2} n$,

$$
\mu\left(\left\{x \in \mathbb{R}^{n} ;|\langle x, \theta\rangle|>C_{\varepsilon} t M\right\}\right)<e^{-c_{\varepsilon} \frac{t^{2}}{\log 4+\varepsilon}(t+1)},
$$

where $C_{\varepsilon}, c_{\varepsilon}>0$ are constants depending only on $\varepsilon$.

Proof. In this proof, $c, C, c^{\prime}$, etc. denote constants that depend solely on $\varepsilon$. Let $2 \leq t \leq$ $\sqrt{n}$, and let $1 \leq i \leq\left\lfloor\log _{2} n\right\rfloor$ be the integer such that $2^{i} \leq t^{2}<2^{i+1}$. Since $\theta / M \in \hat{\hat{T}}_{\varepsilon}(f)$, we have

$$
\int_{\mathbb{R}^{n}} \exp \left(\left\langle\frac{t}{C M(\log t)^{2+\varepsilon}} \theta, y\right\rangle\right) d \mu(y) \leq \int_{\mathbb{R}^{n}} \exp \left(\left\langle\frac{\theta}{M} \cdot \frac{2^{i / 2}}{i^{2+\varepsilon}}, y\right\rangle\right) d \mu(y) \leq e^{2^{i}} \leq e^{t^{2}},
$$

for an appropriate choice of the constant $C>0$. An application of the ChebychevMarkov inequality yields

$$
\mu\left(\left\{x \in \mathbb{R}^{n} ;\langle\theta, x\rangle>2 C M t \log ^{2+\varepsilon} t\right\}\right) \leq \frac{e^{t^{2}}}{e^{2 t^{2}}}=e^{-t^{2}}
$$

for any $2 \leq t \leq \sqrt{n}$. Since $f$ is even, $\hat{T}_{\varepsilon}(f)$ is centrally symmetric, and $-\theta / M \in \hat{T}_{\varepsilon}(f)$. Repeating the above argument for $-\theta$, and substituting $s=t \log ^{2+\varepsilon} t$ in (47), we see that

$$
\mu\left(\left\{x \in \mathbb{R}^{n} ;|\langle\theta, x\rangle|>\tilde{C} M s\right\}\right) \leq e^{-c^{\prime} \frac{s^{2}}{\log ^{4}+2 \varepsilon(s+1)}}
$$

for any $1 \leq s \leq \sqrt{n} \log ^{2+\varepsilon} n$. The lemma is proved. 
Lemma 3.3. Let $n \geq 2$ be an integer, and let $f: \mathbb{R}^{n} \rightarrow[0, \infty)$ be an even, log-concave function with $\int f=1$. For $0<t \leq n$, denote $K=\left\{x \in \mathbb{R}^{n} ; \Upsilon f(x) \leq t\right\}$. Then

$$
\operatorname{v.rad} .\left(K^{\circ}\right)>\frac{c}{\sqrt{t} f(0)^{\frac{1}{n}}},
$$

where $c>0$ is a universal constant.

Proof. Arguing as in the proof of Lemma 2.8, we may clearly assume that $f$ is continuously differentiable. The function $\Upsilon f$ is even, strictly convex, $C^{\infty}$-smooth and $\Upsilon f(0)=0$. Thus, $\Upsilon f$ satisfies the requirements of Lemma 2.3. Let $C_{1}$ denote the constant from Lemma 2.7(iii). Fix $x \in \mathbb{R}^{n}$ such that $\max \left\{C_{1}, 2\right\} x \in K$. Then

$$
\Upsilon\left(C_{1} x\right) \leq t \leq n
$$

Denote $f_{x}(y)=e^{\langle x, y\rangle} f(y)$; this is a log-concave function. By Lemma 2.7(iii),

$$
\sup _{y \in \mathbb{R}^{n}} f_{x}(y)=\sup _{y \in \mathbb{R}^{n}} e^{\langle x, y\rangle} f(y) \leq e^{n} f(0) .
$$

The convex, even function $\Upsilon f$ vanishes at the origin. Hence, $\Upsilon f$ is a nonnegative function, and

$$
\int_{\mathbb{R}^{n}} f_{x}(y) d y=\int_{\mathbb{R}^{n}} e^{\langle x, y\rangle} f(y) d y=\exp \Upsilon f(x) \geq 1 .
$$

Consequently, by (45), (48), (49) and Lemma 3.1,

$$
\operatorname{det} \operatorname{Cov}\left(f_{x}\right)=L_{f_{x}}^{2 n}\left(\frac{\int_{\mathbb{R}^{n}} f_{x}(y) d y}{\sup _{y \in \mathbb{R}^{n}} f_{x}(y)}\right)^{2} \geq \frac{c^{2 n}}{f(0)^{2}} .
$$

By Lemma 2.2, we know that

$$
\operatorname{det} \operatorname{Hess}(\Upsilon f)(x)=\operatorname{det} \operatorname{Cov}\left(f_{x}\right) .
$$

From (50), we deduce that

$$
\operatorname{det} \operatorname{Hess}(\Upsilon f)(x)>\left(\frac{\tilde{c}}{f(0)^{2 / n}}\right)^{n} \text { if } \max \left\{C_{1}, 2\right\} x \in K .
$$

Thus, we may apply Lemma 2.3. In accordance with the conclusion of that lemma,

$$
\text { v. } \operatorname{rad} .(K) \leq C \sqrt{t} f(0)^{\frac{1}{n}} \text {. }
$$

By the Bourgain-Milman inequality [10], we have v.rad. $\left(K^{\circ}\right) \geq\left(C^{\prime} \sqrt{t} f(0)^{1 / n}\right)^{-1}$, which proves the lemma.

For a log-concave function $f: \mathbb{R}^{n} \rightarrow[0, \infty)$ with $0<\int f<\infty$ and for a subspace $E \subset \mathbb{R}^{n}$, we define $\pi_{E}(f): E \rightarrow[0, \infty)$ to be the marginal function

$$
\pi_{E}(f)(x)=\int_{x+E^{\perp}} f(y) d y
$$

where $E^{\perp}$ is the orthogonal complement to $E$ in $\mathbb{R}^{n}$. The Prékopa-Leindler inequality (16) implies that $\pi_{E}(f)$ is also a log-concave function. The following lemma is an immediate consequence of the definitions.

Lemma 3.4. Let $n \geq 1$ be an integer. Suppose $f: \mathbb{R}^{n} \rightarrow[0, \infty)$ is an even, isotropic, log-concave function with $f(0)=\int f=1$. Let $1 \leq k \leq n$ be an integer, and let $E \subset \mathbb{R}^{n}$ be a subspace with $\operatorname{dim}(E)=k$. Then

$$
\left(\pi_{E}(f)(0)\right)^{\frac{1}{k}}=\left(\int_{E^{\perp}} f(x) d x\right)^{\frac{1}{k}}=\frac{L_{\pi_{E}(f)}}{L_{f}} .
$$


Proof. Since the function $f$ is isotropic,

$$
\operatorname{Cov}(f)=\left(\frac{\int f}{f(0)}\right)^{\frac{2}{n}} L_{f}^{2} \mathrm{Id}=L_{f}^{2} \mathrm{Id}
$$

where we used the fact that $1=f(0)=\sup f$ because $f$ is even and log-concave. Consequently, also

$$
\operatorname{Cov}\left(\pi_{E}(f)\right)=L_{f}^{2} \mathrm{Id}
$$

Since $\pi_{E}(f)$ is even and log-concave, we see that $\sup \pi_{E}(f)=\pi_{E}(f)(0)=\int_{E^{\perp}} f$. Using (51) and the definition (45), we deduce that

$$
L_{\pi_{E}(f)}=\left(\frac{\pi_{E}(f)(0)}{\int_{E} \pi_{E}(f)}\right)^{\frac{1}{k}} L_{f}=\left(\pi_{E}(f)(0)\right)^{\frac{1}{k}} L_{f}=\left(\int_{E^{\perp}} f(x) d x\right)^{\frac{1}{k}} L_{f}
$$

as $\int_{E} \pi_{E}(f)=\int_{\mathbb{R}^{n}} f=1$.

For a subspace $E \subset \mathbb{R}^{n}$ we denote by $\operatorname{Proj}_{E}: \mathbb{R}^{n} \rightarrow E$ the orthogonal projection operator onto $E$ in $\mathbb{R}^{n}$.

Lemma 3.5. Let $n \geq 2$, and let $f: \mathbb{R}^{n} \rightarrow[0, \infty)$ be an even, isotropic, log-concave function with $f(0)=\int f=1$. Let $2 \leq k \leq n$ be an integer, and denote $K=\{x \in$ $\left.\mathbb{R}^{n} ; \Upsilon f(x) \leq k\right\}$. Then, for any subspace $F \subset \mathbb{R}^{n}$ with $\operatorname{dim}(F)=\ell \geq k$,

$$
\text { v. } \operatorname{rad} .\left(\operatorname{Proj}_{F}\left(K^{\circ}\right)\right)=\left(\frac{\operatorname{Vol}_{\ell}\left(\operatorname{Proj}_{F}\left(K^{\circ}\right)\right)}{\operatorname{Vol}_{\ell}\left(D^{\ell}\right)}\right)^{\frac{1}{\ell}} \leq C \frac{L_{f}}{\sqrt{k}},
$$

where $C>0$ is a universal constant.

Proof. Fix a subspace $E \subset \mathbb{R}^{n}$ of dimension $k$, and denote $g=\pi_{E}(f)$. From Lemma 3.4, we know that $g(0)^{\frac{1}{k}}=\frac{L_{g}}{L_{f}}$. Note that $L_{g}>c$ by Lemma 3.1. Consequently,

$$
g(0)^{\frac{1}{k}}>\frac{c}{L_{f}}
$$

Set $K_{E}=\{x \in E ; \Upsilon g(x) \leq k\}$. Observe that

$$
\Upsilon f(x)=\Upsilon \pi_{E}(f)(x)=\Upsilon g(x) \text { for } x \in E .
$$

Therefore, $K_{E}=K \cap E$. Recall that $\operatorname{dim}(E)=k$. Lemma 2.8 implies

$$
\text { v. } \operatorname{rad} .(K \cap E)=\text { v. } \operatorname{rad} .\left(K_{E}\right)>c^{\prime} \sqrt{k} g(0)^{\frac{1}{k}} \text {. }
$$

From (52) and (53) we conclude that, for any subspace $E \subset \mathbb{R}^{n}$ of dimension $k$,

$$
\text { v. } \operatorname{rad} .(K \cap E)>\tilde{c} \frac{\sqrt{k}}{L_{f}} .
$$

The subspace $F \subset \mathbb{R}^{n}$ satisfies $\operatorname{dim}(F)=\ell \geq k$. Note that (54) holds true for all the subspaces $E \subset F$ with $\operatorname{dim}(E)=k$. Next we call upon Corollary 3.1 in [17]. By the conclusion of that corollary,

$$
\text { v. } \operatorname{rad} .(K \cap F)>c \frac{\sqrt{k}}{L_{f}} .
$$

Since $(K \cap F)^{\circ}=\operatorname{Proj}_{F}\left(K^{\circ}\right)$, Santaló's inequality and (55) imply that

$$
\text { v. } \operatorname{rad} .\left(\operatorname{Proj}_{F}\left(K^{\circ}\right)\right)<C \frac{L_{f}}{\sqrt{k}} .
$$

Thus, the lemma is proved. 
For $K, T \subset \mathbb{R}^{n}$, the covering number of $K$ by $T$ is defined as

$$
N(K, T)=\min \{\#(A) ; K \subset A+T\},
$$

where $\#(A)$ denotes the cardinality of the set $A$, and + is the Minkowski sum. We will frequently use the following elementary properties of covering numbers. Obviously, $N(A, C) \leq N(A, B) N(B, C)$ for any $A, B, C \subset \mathbb{R}^{n}$. Also, $\operatorname{Vol}_{n}(K) \leq N(K, T) \operatorname{Vol}_{n}(T)$. In addition, for $K_{1}, \ldots, K_{m}, T_{1}, \ldots, T_{m} \subset \mathbb{R}^{n}$,

$$
N\left(K_{1}+\cdots+K_{m}, T_{1}+\cdots+T_{m}\right) \leq \prod_{i=1}^{m} N\left(K_{i}, T_{i}\right) .
$$

Finally, $N(u(K), u(T)) \leq N(K, T)$ whenever $u$ is a linear map.

Let $K \subset \mathbb{R}^{n}$ be a centrally symmetric convex body, and let $\alpha, a, b>0$. An ellipsoid $\mathcal{E} \subset \mathbb{R}^{n}$ is called a "Milman ellipsoid of order $\alpha$ for $K$ with constants $a, b$ " if for any $t>1$,

$$
\max \left\{N(K, a t \mathcal{E}), N(\mathcal{E}, a t K), N\left(K^{\circ}, a t \mathcal{E}^{\circ}\right), N\left(\mathcal{E}^{\circ}, a t K^{\circ}\right)\right\} \leq e^{b \frac{n}{t^{\alpha}}} .
$$

We refer the reader, e.g., to [25] for background on the Milman ellipsoid. A fundamental theorem of Pisier (see [31, §7]) states that, for any centrally symmetric convex body $K \subset \mathbb{R}^{n}$ and any $0<\alpha<2$, there exists a Milman ellipsoid of order $\alpha$ for $K$, with constants that are not larger than $\frac{C}{2-\alpha}$, where $C>0$ is a universal constant.

Next, we apply Lemma 3.5 in order to show that a Milman ellipsoid for the convex set $\left\{x \in \mathbb{R}^{n} ; \Upsilon f(x) \leq k\right\}$ is not too far from a Euclidean ball.

Lemma 3.6. Suppose $A>0,0<\varepsilon \leq 1$. Let $n \geq 4$ be an integer, and let $f: \mathbb{R}^{n} \rightarrow[0, \infty)$ be an even, isotropic, log-concave function with $f(0)=\int f=1$. Suppose that $L_{f}<A$. Let $2 \leq k \leq\left\lfloor\frac{n}{2}\right\rfloor$ be an integer, and denote $K=\left\{x \in \mathbb{R}^{n} ; \Upsilon f(x) \leq k\right\}$. Then for any $1 \leq t \leq c_{A, \varepsilon} \sqrt{\frac{n}{k}}$ we have

$$
N\left(\sqrt{k} K^{\circ}, C_{A, \varepsilon} t D\right)<\exp \left(C_{A, \varepsilon} \cdot \frac{n}{t^{1-\varepsilon}}\right) .
$$

Here $c_{A, \varepsilon}, C_{A, \varepsilon}>0$ are constants depending only on $A$ and $\varepsilon$.

Proof. In this proof we denote by $c, C, c^{\prime}, \tilde{c}$, etc. constants depending only on $A$ and $\varepsilon$. Denote $T=\sqrt{k} K^{\circ}$. The set $T$ is convex and centrally symmetric. By Lemma 3.3,

$$
\text { v. } \operatorname{rad} .(T)>c \text {. }
$$

Let $\alpha=2-\varepsilon \geq 1$. Let $\mathcal{E}$ be a Milman ellipsoid of order $\alpha$ for $T$ with constants $C, C^{\prime}>0$. That is, for any $t \geq 1$,

$$
\max \{N(T, C t \mathcal{E}), N(\mathcal{E}, C t T)\} \leq \exp \left(C^{\prime} \frac{n}{t^{\alpha}}\right) .
$$

We denote by $\lambda_{1} \geq \cdots \geq \lambda_{n}>0$ the lengths of the axes of the $n$-dimensional ellipsoid $\mathcal{E}$. From (58) and from the case of $t=1$ in (59), we conclude that

$$
c<\mathrm{v} \cdot \operatorname{rad} .(T) \leq N(T, C \mathcal{E})^{\frac{1}{n}} \text { v. } \operatorname{rad} .(C \mathcal{E}) \leq \hat{C} \text { v. } \operatorname{rad} .(\mathcal{E}) .
$$

Note that v.rad. $(\mathcal{E})=\left(\prod_{i=1}^{n} \lambda_{i}\right)^{\frac{1}{n}}$. Hence, $(60)$ implies that

$$
\left(\prod_{i=1}^{n} \lambda_{i}\right)^{\frac{1}{n}}>c
$$

Next, set $m=\left\lfloor\frac{n}{2}\right\rfloor$, and let $F$ denote the subspace spanned by the $m$ longest axes of the ellipsoid $\mathcal{E}$. Projecting (59) to the subspace $F$, and substituting $t=1$, we get

$$
N\left(\operatorname{Proj}_{F}(\mathcal{E}), C \operatorname{Proj}_{F}(T)\right) \leq e^{C^{\prime} n} .
$$


Hence, v. $\operatorname{rad} .\left(\operatorname{Proj}_{F}(\mathcal{E})\right) \leq \hat{C}$ v.rad. $\left(\operatorname{Proj}_{F}(T)\right)$. We combine this estimate with Lemma 3.5 to obtain

$$
\left(\prod_{i=1}^{m} \lambda_{i}\right)^{\frac{1}{m}}=\text { v. rad. }\left(\operatorname{Proj}_{F}(\mathcal{E})\right) \leq C \text { v. } \operatorname{rad} .\left(\operatorname{Proj}_{F}\left(\sqrt{k} K^{\circ}\right)\right)<C^{\prime} L_{f}<\tilde{C}
$$

(recall that $L_{f}<A$ ). From (61) and (62) we deduce that

$$
\prod_{i=m+1}^{n} \lambda_{i}=\left(\prod_{i=1}^{n} \lambda_{i}\right) \cdot\left(\prod_{i=1}^{m} \lambda_{i}\right)^{-1}>c^{n} \cdot \bar{c}^{m}>\tilde{c}^{n-m} .
$$

Since $\lambda_{i}$ is a monotone nonincreasing sequence, we have

$$
\lambda_{\left\lfloor\frac{n}{2}\right\rfloor} \geq \lambda_{m+1} \geq\left(\prod_{i=m+1}^{n} \lambda_{i}\right)^{\frac{1}{n-m}}>\tilde{c} .
$$

Next, let $\ell$ be an integer with $k \leq \ell \leq \frac{n}{2}$. Now, let $F$ denote the $\ell$-dimensional subspace spanned by the $\ell$ longest axes of the ellipsoid $\mathcal{E}$. Projecting (59) to $F$, we see that, for any $t>1$,

$$
N\left(\operatorname{Proj}_{F}(\mathcal{E}), C t \operatorname{Proj}_{F}\left(\sqrt{k} K^{\circ}\right)\right)<\exp \left(C^{\prime} \frac{n}{t^{\alpha}}\right) .
$$

Setting $t=\left(\frac{n}{\ell}\right)^{\frac{1}{\alpha}}$ in (64), we deduce that

$$
\left(\prod_{i=1}^{\ell} \lambda_{i}\right)^{\frac{1}{\ell}}=\text { v.rad. }\left(\operatorname{Proj}_{F}(\mathcal{E})\right)<C\left(\frac{n}{\ell}\right)^{\frac{1}{\alpha}} \text { v.rad. }\left(\operatorname{Proj}_{F}\left(\sqrt{k} K^{\circ}\right)\right) \text {. }
$$

Lemma 3.5, combined with (65), gives

$$
\left(\prod_{i=1}^{\ell} \lambda_{i}\right)^{\frac{1}{\ell}}<C A\left(\frac{n}{\ell}\right)^{\frac{1}{\alpha}}<\tilde{C}\left(\frac{n}{\ell}\right)^{\frac{1}{\alpha}}
$$

Since $\lambda_{i}$ is a monotone nonincreasing sequence, (66) implies that

$$
\lambda_{\ell} \leq\left(\prod_{i=1}^{\ell} \lambda_{i}\right)^{\frac{1}{\ell}}<C\left(\frac{n}{\ell}\right)^{\frac{1}{\alpha}}
$$

Hence,

$$
\prod_{i=1}^{n} \max \left\{\frac{1}{C}\left(\frac{\ell}{n}\right)^{\frac{1}{\alpha}} \lambda_{i}, 1\right\}=\prod_{i=1}^{\ell} \max \left\{\frac{1}{C}\left(\frac{\ell}{n}\right)^{\frac{1}{\alpha}} \lambda_{i}, 1\right\} .
$$

We use (67) together with (63) and (66) to obtain

$$
\begin{aligned}
\prod_{i=1}^{n} \max \left\{\frac{1}{C}\left(\frac{\ell}{n}\right)^{\frac{1}{\alpha}} \lambda_{i}, 1\right\} & \leq \prod_{i=1}^{\ell} \max \left\{\frac{1}{C}\left(\frac{\ell}{n}\right)^{\frac{1}{\alpha}} \lambda_{i}, \frac{1}{\tilde{c}} \lambda_{i}\right\} \\
& \leq\left(\frac{1}{\min \{C, \tilde{c}\}}\right)^{\ell} \prod_{i=1}^{\ell} \lambda_{i} \leq\left(C^{\prime}\left(\frac{n}{\ell}\right)^{\frac{1}{\alpha}}\right)^{\ell}
\end{aligned}
$$

(recall that $\ell \leq\left\lfloor\frac{n}{2}\right\rfloor$ ). Next, we shall use standard estimates for the number of Euclidean balls needed to cover an ellipsoid (see, e.g., Remark 5.15 in [31]). Recall that $\lambda_{1}, \ldots, \lambda_{n}$ are the lengths of the axes of the ellipsoid $\mathcal{E}$. Then, for any $r>0$ we have $N\left(\mathcal{E}, 4 r D^{n}\right) \leq$ $\prod_{i=1}^{n} \max \left\{\lambda_{i} / r, 1\right\}$. From (68) we thus conclude that

$$
N\left(\mathcal{E}, \tilde{C}\left(\frac{n}{\ell}\right)^{\frac{1}{\alpha}} D^{n}\right)<\left(C^{\prime}\left(\frac{n}{\ell}\right)^{\frac{1}{\alpha}}\right)^{\ell}
$$


for any $k \leq \ell \leq\left\lfloor\frac{n}{2}\right\rfloor$. Next, suppose $t>C$ is such that $t^{\alpha} \log t \leq \frac{n}{k}$. Using (59) followed by the case of $\ell=\frac{n}{t^{\alpha} \log t}$ in (69), we get

$$
\begin{aligned}
N\left(T, C t^{2} \log t D\right) & \leq N\left(T, C^{\prime} t \mathcal{E}\right) N\left(C^{\prime} t \mathcal{E}, C t^{2} \log ^{\frac{1}{\alpha}} t D\right) \\
& \leq e^{c \frac{n}{t^{\alpha}}} \cdot\left(\tilde{C} t \log ^{\frac{1}{\alpha}} t\right)^{\frac{n}{t^{\alpha} \log t}}<e^{\hat{C} \frac{n}{t^{\alpha}}}
\end{aligned}
$$

(recall that $\alpha=2-\varepsilon \geq 1$ ). Now (70) implies that if $\bar{C} \leq t \leq c \sqrt{\frac{n}{k}}$, then

$$
N(T, C t D) \leq \exp \left(C^{\prime} n \frac{\log t}{t^{1-\frac{\varepsilon}{2}}}\right)<\exp \left(\tilde{C} \frac{n}{t^{1-\varepsilon}}\right)
$$

(note that $c \sqrt{\frac{n}{k}} \leq C\left(\frac{n}{k}\right)^{\frac{1}{\alpha}}\left(\log \frac{n}{k}\right)^{-1}$ ). By selecting appropriate constants $C, \tilde{C}$ in $(71)$, we see that $(71)$ is true also for $1 \leq t \leq \bar{C}$. The proof is thus complete.

Recall the definition (46) of the set $\hat{T}_{\varepsilon}(f)$.

Lemma 3.7. Suppose $0<\varepsilon \leq 1$ and $A>0$. Let $n \geq 4$ be an integer, and let $f: \mathbb{R}^{n} \rightarrow$ $[0, \infty)$ be an even, log-concave function with $\int f=1$. Suppose that $L_{f}<A$. Then

$$
\text { v. } \operatorname{rad} .\left(\hat{T}_{\varepsilon}(f)\right)>c_{A, \varepsilon} f(0)^{\frac{1}{n}} .
$$

Here, $c_{A, \varepsilon}>0$ is a constant depending only on $A$ and $\varepsilon$.

Proof. In this proof, $c, C, c^{\prime}$, etc. denote constants that depend solely on $A$ and $\varepsilon$. First, suppose that $f(0)=1$ and that $f$ is isotropic. For $t>0$, denote

$$
K_{t}=\left\{x \in \mathbb{R}^{n} ; \Upsilon f(x) \leq t\right\} .
$$

Dualizing (46), we get

$$
\left(\hat{T}_{\varepsilon}(f)\right)^{\circ}=\operatorname{conv}\left(\bigcup_{i=1}^{\left\lfloor\log _{2} n\right\rfloor} \frac{2^{i / 2}}{i^{2+\varepsilon}} K_{2^{i}}^{\circ}\right),
$$

where conv denotes convex hull. By the Bourgain-Milman inequality [10], it suffices to prove that

$$
\text { v. } \operatorname{rad} .\left(\operatorname{conv} \bigcup_{i=1}^{\left\lfloor\log _{2} n\right\rfloor} \frac{2^{i / 2}}{i^{2+\varepsilon}} K_{2^{i}}^{\circ}\right)<C .
$$

For any centrally symmetric convex bodies $\Omega_{1}, \ldots, \Omega_{k} \subset \mathbb{R}^{n}$, we know that $\operatorname{conv}\left(\Omega_{1} \cup\right.$ $\left.\cdots \cup \Omega_{k}\right) \subset \Omega_{1}+\cdots+\Omega_{k}$. Consequently, it suffices to prove that

$$
\text { v. } \operatorname{rad}\left(\sum_{i=1}^{\left\lfloor\log _{2} n\right\rfloor} \frac{2^{i / 2}}{i^{2+\varepsilon}} K_{2^{i}}^{\circ}\right)<C .
$$

We define $c_{1}$ to be the largest possible number such that the following two requirements are fulfilled:

$$
\begin{gathered}
i^{1+\varepsilon / 2} \leq c_{A, \varepsilon / 6} \sqrt{\frac{n}{2^{i}}} \text { whenever } 1 \leq 2^{i} \leq \frac{c_{1} n}{\log ^{4} n}, \\
\text { and } c_{1} \leq \min \left\{c_{A, 1 / 3}^{2}, 1\right\},
\end{gathered}
$$

where $c_{A, \varepsilon / 6}$ and $c_{A, 1 / 3}$ are the constants from Lemma 3.6. Then $c_{1}$ is a constant depending only on $A$ and $\varepsilon$. We introduce the functions $\log ^{(k)} t=\max \left\{\left(\log \log ^{(k-1)} t\right)^{4}, c_{1}\right\}$ recursively, starting with $\log ^{(0)} t=t$. Next, we divide the Minkowski sum in (72) into parts. We set

$$
S_{1}=\sum_{i=1}^{\left\lfloor\log _{2} \frac{c_{1} n}{\log ^{4} n}\right\rfloor} \frac{2^{i / 2}}{i^{2+\varepsilon}} K_{2^{i}}^{\circ}=\sum_{i=1}^{\left\lfloor\log _{2} \frac{c_{1} n}{\log ^{(1)} n}\right\rfloor} \frac{2^{i / 2}}{i^{2+\varepsilon}} K_{2^{i}}^{\circ}
$$


and for any $\mu \geq 2$ such that $\log ^{(\mu-1)} n>c_{1}$, we define

$$
S_{\mu}=\sum_{i=\left\lfloor\log _{2} \frac{c_{1 n}}{\log (\mu-1)_{n}}\right\rfloor+1}^{\left\lfloor\log _{2} \frac{c_{1} n}{\log (\mu)_{n}}\right\rfloor} \frac{2^{i / 2}}{i^{2+\varepsilon}} K_{2^{i}}^{\circ}
$$

(where an empty Minkowski sum, if one occurs, equals the empty set). Thus, to establish (72), it suffices to prove that

$$
\text { v. rad. }\left(\sum_{\mu \geq 1} S_{\mu}\right) \leq C .
$$

We begin with estimating $S_{1}$, the most significant term. Fix a positive integer $i$ such that $2^{i} \leq \frac{c_{1} n}{\log ^{4} n}$. Because of (73), we may apply Lemma 3.6 for $k=2^{i}$, for the body $K_{2^{i}}$, for $\varepsilon / 6$, and for $t=i^{1+\varepsilon / 2}$. By the conclusion of that lemma,

$$
N\left(2^{i / 2} K_{2^{i}}^{\circ} C i^{1+\varepsilon / 2} D\right) \leq \exp \left(\frac{C n}{i^{(1+\varepsilon / 2)(1-\varepsilon / 6)}}\right) \leq \exp \left(\frac{C^{\prime} n}{i^{1+\varepsilon / 4}}\right) .
$$

Equivalently,

$$
N\left(\frac{2^{i / 2}}{i^{2+\varepsilon}} K_{2^{i}}^{\circ}, \frac{C}{i^{1+\varepsilon / 2}} D\right) \leq \exp \left(\frac{C^{\prime} n}{i^{1+\varepsilon / 4}}\right) .
$$

The definition of $S_{1}$ and estimates (78) and (56) imply a certain bound on the quantity $N\left(S_{1}, C \sum_{i} \frac{1}{i^{1+\varepsilon / 2}} D\right)$. Note that $\sum_{i=1}^{\infty} \frac{1}{i^{1+\varepsilon / 2}}<C^{\prime}$. By (56), (75), and (78),

$$
N\left(S_{1}, C^{\prime} D\right) \leq \exp \left(\sum_{i} \tilde{C} \frac{n}{i^{1+\varepsilon / 4}}\right)<e^{C^{\prime \prime} n} .
$$

Next, we analyze $S_{\mu}$ for any relevant $\mu \geq 2$. Pick a positive integer $i$ such that $\frac{c_{1} n}{\log ^{(\mu-1)} n}<$ $2^{i} \leq \frac{c_{1} n}{\log (\mu)}$. We may use Lemma 3.6 for $k=2^{i}$, for the body $K_{2^{i}}$, for $\varepsilon=\frac{1}{3}$ and for $t=\sqrt{\log ^{(\mu)} n}$ (note that (74) gives $t \leq c_{A, 1 / 3} \sqrt{n / k}$, as required). By the conclusion of that lemma,

$$
N\left(2^{i / 2} K_{2^{i}}^{\circ}, C \sqrt{\log ^{(\mu)} n D}\right) \leq \exp \left(\frac{C^{\prime} n}{\left(\log ^{(\mu)} n\right)^{1 / 3}}\right) .
$$

Equivalently (note that $c \log n \leq i \leq C \log n$ whenever $2^{i} \geq \frac{c_{1} n}{\log ^{(1)} n}$ ),

$$
\left.N\left(\frac{2^{i / 2}}{i^{2+\varepsilon}} K_{2^{i}}^{\circ}, C \frac{\sqrt{\log ^{(\mu)} n}}{\log ^{2+\varepsilon} n} D\right) \leq \exp \left(\frac{C^{\prime} n}{(\log (\mu)} n\right)^{1 / 3}\right) .
$$

The number of summands in $S_{\mu}$ is bounded by

$$
C \log \left(\log ^{(\mu-1)} n\right)=C\left(\log ^{(\mu)} n\right)^{1 / 4},
$$

and each of these summands satisfies (80). Consequently, (80), (76) and (56) imply that, for any $\mu \geq 2$,

$$
N\left(S_{\mu}, C^{\prime} \frac{\left(\log { }^{(\mu)} n\right)^{1 / 4} \sqrt{\log ^{(\mu)} n}}{\log ^{2+\varepsilon} n} D\right) \leq \exp \left(\tilde{C} n \frac{\left(\log ^{(\mu)} n\right)^{1 / 4}}{\left(\log ^{(\mu)} n\right)^{1 / 3}}\right) .
$$

Next, we estimate the number of balls needed to cover $\sum_{\mu \geq 2} S_{\mu}$. By (81) and (56),

$$
N\left(\sum_{\mu \geq 2} S_{\mu}, C^{\prime}\left(\sum_{\mu \geq 2} \frac{\left(\log ^{(\mu)} n\right)^{3 / 4}}{\log ^{2+\varepsilon} n}\right) D\right) \leq \exp \left(\tilde{C} n \sum_{\mu \geq 2} \frac{1}{\left(\log ^{(\mu)} n\right)^{1 / 12}}\right) .
$$


Both numerical sums in (82) are bounded by a universal constant. Thus, (82) implies the simpler estimate

$$
N\left(\sum_{\mu \geq 2} S_{\mu}, \hat{C} D\right) \leq e^{C^{\prime \prime} n} .
$$

Estimates (79) and (83), together with (56), imply that $N\left(\sum_{\mu \geq 1} S_{\mu}, C^{\prime} D\right) \leq e^{C n}$. Hence,

$$
\text { v. } \operatorname{rad} .\left(\sum_{\mu \geq 1} S_{\mu}\right) \leq C
$$

and (77) is proved. This completes the proof in the case where $f(0)=1$ and $f$ is isotropic.

The general case easily follows. Suppose $f: \mathbb{R}^{n} \rightarrow[0, \infty)$ is an even, log-concave function with $\int f=1$. Let $u: \mathbb{R}^{n} \rightarrow \mathbb{R}^{n}$ be a linear map such that the function $f_{u}(x)=$ $(\operatorname{det} u) \cdot f(u(x))$ satisfies $f_{u}(0)=\int f_{u}=1$ and $f_{u}$ is isotropic. Clearly $f(0)=\frac{1}{\operatorname{det} u}$. Moreover, by (29),

$$
\text { v. } \operatorname{rad} .\left(\hat{T}_{\varepsilon}(f)\right)=(\operatorname{det} u)^{-\frac{1}{n}} \text { v.rad. }\left(\hat{T}_{\varepsilon}\left(f_{u}\right)\right)=f(0)^{\frac{1}{n}} \text { v.rad. }\left(\hat{T}_{\varepsilon}\left(f_{u}\right)\right) .
$$

Note also that $L_{f_{u}}=L_{f}<A$. Thus, we are in the case we have already treated, and, in accordance with the above discussion, v. $\operatorname{rad} .\left(\hat{T}_{\varepsilon}\left(f_{u}\right)\right)>C$. The lemma now follows from (84).

\section{$\S 4$. Proof of the Main Result}

In this section we complete the proofs of Theorem 1.1 and Theorem 1.3. Our first step is to remove the assumption that $L_{f}$ is bounded.

Lemma 4.1. Let $n \geq 1$ be an integer, and let $f: \mathbb{R}^{n} \rightarrow[0, \infty)$ be an isotropic, even, log-concave function with $\int f=1$. Let $M>0$ be such that $\operatorname{Cov}(f)=M^{2}$ Id. Denote $g(x)=\frac{1}{\left(2 \pi M^{2}\right)^{n / 2}} \exp \left(-\frac{|x|^{2}}{2 M^{2}}\right)$. Let $f^{\prime}=f * g$, the convolution of $f$ and $g$. Then

$$
L_{f^{\prime}} \leq 1 \text { and } f^{\prime}(0)^{\frac{1}{n}} \geq \frac{c}{M}
$$

where $c>0$ is a universal constant.

Proof. Since both $f$ and $g$ are even and log-concave, so is their convolution $f^{\prime}$. Furthermore, by our assumptions, $\operatorname{Cov}(f)=\operatorname{Cov}(g)=M^{2}$ Id. Consequently,

$$
\operatorname{Cov}\left(f^{\prime}\right)=\operatorname{Cov}(f * g)=\operatorname{Cov}(f)+\operatorname{Cov}(g)=2 M^{2} \mathrm{Id} .
$$

Next, note that

$$
f^{\prime}(0)=\int_{\mathbb{R}^{n}} f(x) g(-x) d x \leq \frac{1}{\left(2 \pi M^{2}\right)^{n / 2}} \int_{\mathbb{R}^{n}} f(x) d x=\left(\frac{1}{\sqrt{2 \pi} M}\right)^{n} .
$$

Clearly $\int f^{\prime}=1$. By (85), (86) and the definition (45),

$$
L_{f^{\prime}}=\left(\frac{\sup f^{\prime}}{\int f^{\prime}}\right)^{\frac{1}{n}} \operatorname{det} \operatorname{Cov}\left(f^{\prime}\right)^{\frac{1}{2 n}}=f^{\prime}(0)^{\frac{1}{n}} \sqrt{2} M \leq \frac{1}{\sqrt{\pi}} \leq 1,
$$

where we have used once again the fact that any even, log-concave function attains its maximum at the origin. By Lemma 3.1, we know that $L_{f^{\prime}}>c$. Since $f^{\prime}$ is isotropic, (85) and the definition (45) imply that

$$
f^{\prime}(0)^{\frac{1}{n}} \geq \frac{c}{\operatorname{det} \operatorname{Cov}\left(f^{\prime}\right)^{\frac{1}{2 n}}} \geq \frac{c^{\prime}}{M} .
$$

The proof is complete. 
Lemma 4.2. Let $0<\varepsilon \leq 1$, let $n \geq 4$ be an integer, and let $f: \mathbb{R}^{n} \rightarrow[0, \infty)$ be an even, log-concave function with $\int f=1$. Denote by $\mu$ the measure whose density is $f$. Then

$$
\text { v.rad. }\left(\hat{T}_{\varepsilon}(f)\right)>c_{\varepsilon} \frac{f(0)^{\frac{1}{n}}}{L_{f}} .
$$

Furthermore, there exists a nonzero linear functional $\varphi: \mathbb{R}^{n} \rightarrow \mathbb{R}$ such that

$$
\mu\left(\left\{x \in \mathbb{R}^{n} ;|\varphi(x)|>t\|\varphi\|_{L_{1}(\mu)}\right\}\right)<e^{-c_{\varepsilon} \frac{t^{2}}{\log ^{4}+\varepsilon(t+1)}}
$$

for any $1 \leq t \leq \sqrt{n} \log ^{2+\varepsilon / 2} n$. Here $c_{\varepsilon}>0$ is a constant depending only on $\varepsilon$.

Proof. In this proof, $c, C, c^{\prime}$, etc. denote constants that depend solely on $\varepsilon$. As we argued before, we may replace $f$ with $f \circ u$, for any $u \in S L_{n}(\mathbb{R})$, without altering the validity of the conclusions of the lemma. We thus replace $f$ with an appropriate $f \circ u$, and assume from now on that $f$ is isotropic.

Let $M=f(0)^{-\frac{1}{n}} L_{f}$. Then $\operatorname{Cov}(f)=M^{2}$ Id. Denote $g(x)=\frac{1}{\left(2 \pi M^{2}\right)^{n / 2}} \exp \left(-\frac{|x|^{2}}{2 M^{2}}\right)$, and set

$$
f^{\prime}=f * g
$$

Then $f^{\prime}$ is an even, log-concave function with $\int f^{\prime}=1$. Furthermore, $L_{f^{\prime}} \leq 1$ and $f^{\prime}(0)^{\frac{1}{n}} \geq c / M$, by Lemma 4.1. We invoke Lemma 3.7 for $f^{\prime}, \varepsilon$ and $A=1$. By the conclusion of that lemma,

$$
\text { v. } \operatorname{rad} .\left(\hat{T}_{\varepsilon}\left(f^{\prime}\right)\right)>\frac{c}{M}=c \frac{f(0)^{\frac{1}{n}}}{L_{f}} .
$$

Note that $\Upsilon\left(f^{\prime}\right)=\Upsilon(f)+\Upsilon(g) \geq \Upsilon(f)$. Consequently, we have $\hat{T}_{\varepsilon}\left(f^{\prime}\right) \subset \hat{T}_{\varepsilon}(f)$, whence

$$
\text { v. } \operatorname{rad} .\left(\hat{T}_{\varepsilon}(f)\right)>c \frac{f(0)^{\frac{1}{n}}}{L_{f}} .
$$

This completes the proof of the first part of the lemma. It remains to prove the second part. To that end, denote

$$
T=\left\{x \in \mathbb{R}^{n} ; \Upsilon f(x) \leq \log 2\right\} .
$$

Suppose $x \in T$. Since $f$ is even, we see that

$$
\int_{\mathbb{R}^{n}}\langle x, y\rangle^{2} f(y) d y=2 \int_{\left\{y \in \mathbb{R}^{n} ;\langle x, y\rangle \geq 0\right\}}\langle x, y\rangle^{2} f(y) d y \leq 4 \int_{\mathbb{R}^{n}} e^{\langle x, y\rangle} f(y) d y \leq 4 \log 2,
$$

where we have used the fact that $t^{2} \leq 2 e^{t}$ for $t>0$. Consequently, $x \in T$ implies $\langle\operatorname{Cov}(f) x, x\rangle \leq 4 \log 2$. We conclude that

$$
\text { v. } \operatorname{rad} .(T) \leq \sqrt{4 \log 2} \frac{1}{\operatorname{det} \operatorname{Cov}(f)^{\frac{1}{2 n}}}=C \frac{f(0)^{1 / n}}{L_{f}},
$$

where the last relation follows from the definition (45). Comparing (89) with (91), we see that

$$
\hat{T}_{\varepsilon}(f) \not \subset c^{\prime} T .
$$

The sets $\hat{T}_{\varepsilon}(f)$ and $T$ are convex and centrally symmetric. In particular, there exists $0 \neq x \in \mathbb{R}^{n}$ such that

$$
\forall s \in \mathbb{R}, \quad s x \in T \Rightarrow c^{\prime} s x \in \hat{T}_{\varepsilon}(f) .
$$

We fix $0 \neq x \in \mathbb{R}^{n}$ that satisfies (92). Let

$$
N=\|\langle\cdot, x\rangle\|_{L_{1}(\mu)}=\int|\langle x, y\rangle| f(y) d y .
$$


It is well known that

$$
\mu\left(\left\{y \in \mathbb{R}^{n} ;|\langle x, y\rangle| \leq N\right\}\right) \geq c
$$

for some universal constant $c>0$ (see, e.g., $[3, \S 3]$ ). Recall Borell's lemma, the $\psi_{1}$ estimate (17). From (17) and (93),

$$
\int_{\mathbb{R}^{n}} \exp \left(\frac{\langle x, y\rangle}{C N}\right) f(y) d y \leq 2 .
$$

Thus, $\frac{x}{C N} \in T$, by the definition (90). By (92),

$$
\frac{x}{C^{\prime} N} \in \hat{T}_{\varepsilon}(f) \text {. }
$$

We may apply Lemma 3.2, based on (95). We conclude that

$$
\mu\left(\left\{y \in \mathbb{R}^{n} ;|\langle x, y\rangle|>C N s\right\}\right) \leq e^{-c^{\prime} \frac{t^{2}}{\log ^{4+\varepsilon}(t+1)}}
$$

for any $1 \leq t \leq \sqrt{n} \log ^{2+\varepsilon / 2} n$. In view of (93) and (94), it is apparent that (96) is equivalent to the desired estimate (87). This completes the proof.

Next, we remove the assumption that the functions are even. First, we prove a result on general log-concave densities.

Theorem 4.3. Let $0<\varepsilon \leq 1$, let $n \geq 1$ be an integer, and let $f: \mathbb{R}^{n} \rightarrow[0, \infty)$ be a log-concave function with $\int f=1$. Denote by $\mu$ the measure whose density is $f$. Then there exists a nonzero linear functional $\varphi: \mathbb{R}^{n} \rightarrow \mathbb{R}$ such that

$$
\mu\left(\left\{x \in \mathbb{R}^{n} ;|\varphi(x)|>t\|\varphi\|_{L_{1}(\mu)}\right\}\right)<e^{-c_{\varepsilon} \frac{t^{2}}{\log ^{4}+\varepsilon}(t+1)}
$$

for any $1 \leq t \leq \sqrt{n} \log ^{2+\varepsilon / 2} n$, where $c_{\varepsilon}>0$ is a constant depending only on $\varepsilon$.

Proof. In this proof, $c, C, c^{\prime}, \tilde{c}$, etc. represent constants depending only on $\varepsilon$. Let $f^{\prime}(x)=$ $f(-x)$, and consider the convolution $g=f * f^{\prime}$. The function $g: \mathbb{R}^{n} \rightarrow[0, \infty)$ is an even, $\log$-concave function with $\int g=1$. Denote by $\nu$ the measure whose density is $g$. We may suppose that $n \geq 4$ (if $n \leq 3$, then, by (94), an appropriate constant $c_{\varepsilon}$ may be selected so that (97) is trivially true). By Lemma 4.2, there exists a nonzero linear functional $\varphi: \mathbb{R}^{n} \rightarrow \mathbb{R}$ such that

$$
\nu\left(\left\{x \in \mathbb{R}^{n} ;|\varphi(x)|>t\|\varphi\|_{L_{1}(\nu)}\right\}\right)<e^{-c \frac{t^{2}}{\log ^{4}+\varepsilon}(t+1)}
$$

for any $1 \leq t \leq \sqrt{n} \log ^{2+\varepsilon / 2} n$. Let $M>0$ be such that

$$
\mu\left(\left\{x \in \mathbb{R}^{n} ;|\varphi(x)| \leq M\right\}\right)=\frac{2}{3} .
$$

By Borell's lemma (17), we know that $c\|\varphi\|_{L_{2}(\mu)} \leq M$. Consequently,

$$
M \geq c\|\varphi\|_{L_{2}(\mu)} \geq c \sqrt{\operatorname{Var}_{\mu}(\varphi)}=c \sqrt{\frac{\operatorname{Var}_{\nu}(\varphi)}{2}}=\frac{c}{\sqrt{2}}\|\varphi\|_{L_{2}(\nu)} \geq \frac{c}{\sqrt{2}}\|\varphi\|_{L_{1}(\nu)},
$$

where, for any probability measure $\rho$ on $\mathbb{R}^{n}$, we write

$$
\operatorname{Var}_{\rho}(\varphi)=\int_{\mathbb{R}^{n}} \varphi^{2}(y) d \rho(y)-\left(\int_{\mathbb{R}^{n}} \varphi(y) d \rho(y)\right)^{2},
$$

the variance of $\varphi$ with respect to the measure $\rho$. Let $X$ and $Y$ be independent random vectors that are distributed according to the densities $f$ and $f^{\prime}$, respectively. Then the 
random vector $X+Y$ is distributed according to $\nu$. Note that $\operatorname{Prob}\{|\varphi(Y)| \leq M\}=\frac{2}{3}$. Consequently, by (99), for any $1 \leq t \leq \sqrt{n} \log ^{2+\varepsilon / 2} n$,

$$
\begin{aligned}
& \frac{2}{3} \operatorname{Prob}\{|\varphi(X)| \geq(t+1) M\} \\
& \quad=\operatorname{Prob}\{|\varphi(X)| \geq(t+1) M,|\varphi(Y)| \leq M\} \leq \operatorname{Prob}\{|\varphi(X+Y)| \geq t M\} \\
& \quad \leq \operatorname{Prob}\left\{|\varphi(X+Y)| \geq c t\|\varphi\|_{L_{1}(\nu)}\right\} \leq e^{-\tilde{c} \frac{t^{2}}{\log ^{4}+\varepsilon(t+1)}},
\end{aligned}
$$

where we used (98) for the last inequality. From (100) we deduce (97), for $C<t<$ $c \sqrt{n} \log ^{2+\varepsilon / 2} n$. Recall that $\mu\left(\left\{x \in \mathbb{R}^{n} ;|\varphi(x)| \geq\|\varphi\|_{L^{1}(\mu)}\right\}\right) \leq 1-c$ (see, e.g., the methods in $[3, \S 3])$. Thus, we may adjust the constants so that (97) will be true for all $1 \leq t \leq \sqrt{n} \log ^{2+\varepsilon / 2} n$. The proof is complete.

We still need to take care of the range $t \geq C \sqrt{n} \log ^{2+\varepsilon / 2} n$. The following lemma serves this purpose.

Lemma 4.4. Let $\alpha, A>0$, let $n \geq \frac{2}{A}$ be an integer, and let $f: \mathbb{R}^{n} \rightarrow[0, \infty)$ be an $\alpha n$-concave function with $\int f=1$ whose barycenter lies at the origin. For $x \in \mathbb{R}^{n}$ and $b>0$, we denote $K=\left\{y \in \mathbb{R}^{n} ;|\langle x, y\rangle| \geq b\right\}$. Suppose that

$$
\int_{K} f(x) d x \leq e^{-A n} \text {. }
$$

Then

$$
\sup _{y \in \operatorname{Supp}(f)}|\langle x, y\rangle| \leq C_{A, \alpha} b,
$$

where $C_{A, \alpha}>0$ is a constant depending solely on $A$ and $\alpha$, and as usual, $\operatorname{Supp}(f)$ is the closure of the set $\left\{x \in \mathbb{R}^{n} ; f(x) \neq 0\right\}$.

Proof. For $t \in \mathbb{R}$, denote

$$
\psi(t)=\left(\int_{\left\{y \in \mathbb{R}^{n} ;\langle x, y\rangle \geq t\right\}} f(y) d y\right)^{\frac{1}{(\alpha+1) n}} .
$$

By (18), the function $\psi$ is concave on its support. The function $f$ is log-concave, $\int f=1$, and the barycenter of $f$ lies at the origin. It is well known that a hyperplane through the origin divides $\mathbb{R}^{n}$ into two half-spaces on each of which the integral of $f$ is not smaller than $\frac{1}{e}$. This fact, essentially going back to Grünbaum and to Hammer (see [15]), was proved, e.g., in [3, Lemma 3.3]. Thus, we know that $\psi(0) \geq\left(\frac{1}{e}\right)^{1 /(\alpha+1) n}$. Our assumptions imply that

$$
\psi(0) \geq\left(\frac{1}{e}\right)^{\frac{1}{(\alpha+1) n}} \geq e^{-\frac{A}{2(\alpha+1)}}, \quad \psi(b) \leq e^{-\frac{A}{\alpha+1}} .
$$

Since $\psi$ is concave on its support, (101) implies the relation

$$
\psi\left(\left(1-e^{-\frac{A}{2(\alpha+1)}}\right)^{-1} b\right)=0 .
$$

Thus, $\operatorname{Supp}(f) \subset\left\{y \in \mathbb{R}^{n} ;\langle x, y\rangle \leq C_{A, \alpha} b\right\}$ for $C_{A, \alpha}=\left(1-e^{-\frac{A}{2(\alpha+1)}}\right)^{-1}$. Repeating the argument for $-x$ in place of $x$, we prove the lemma.

Lemma 4.5. Let $0<\varepsilon \leq 1, \alpha>0$, let $n \geq 1$ be an integer, and let $f: \mathbb{R}^{n} \rightarrow[0, \infty)$ be an even, $\alpha$-concave function with $\int f=1$. Denote by $\mu$ the measure whose density is $f$. Then there exists a nonzero linear functional $\varphi: \mathbb{R}^{n} \rightarrow \mathbb{R}$ such that

$$
\mu\left(\left\{x \in \mathbb{R}^{n} ;|\varphi(x)|>t\|\varphi\|_{L_{1}(\mu)}\right\}\right)<e^{-c_{\varepsilon, \alpha} \frac{t^{2}}{\log ^{4}+\varepsilon(t+1)}}
$$

for any $t \geq 1$, where $c_{\varepsilon, \alpha}>0$ is a constant depending only on $\varepsilon$ and $\alpha$. 
Proof. In this proof, $c_{1}, c_{2}, C, C^{\prime}, \tilde{C}$, etc. denote positive constants that depend solely on $\varepsilon$ and $\alpha$. An $\alpha n$-concave function is clearly log-concave. First, suppose that $n \geq 4$. Thus, we may apply Lemma 4.2. By the conclusion of that lemma, there exists a nonzero linear functional $\varphi: \mathbb{R}^{n} \rightarrow \mathbb{R}$ such that

$$
\mu\left(\left\{x \in \mathbb{R}^{n} ;|\varphi(x)|>t\|\varphi\|_{L_{1}(\mu)}\right\}\right) \leq e^{-c \frac{t^{2}}{\log 4+\varepsilon(t+1)}}
$$

for any $1 \leq t \leq \sqrt{n} \log ^{2+\varepsilon / 2} n$. Substituting $t=\sqrt{n} \log ^{2+\varepsilon / 2} n$ in (103), we obtain

$$
\mu\left(\left\{x \in \mathbb{R}^{n} ;|\varphi(x)|>\sqrt{n}(\log n)^{2+\varepsilon / 2}\|\varphi\|_{L_{1}(\mu)}\right\}\right) \leq e^{-c_{1} n} .
$$

Consider first the case of $n \geq \frac{2}{c_{1}}$, where $c_{1}$ is the constant in (104). We may apply Lemma 4.4, based on (104), and deduce that

$$
\mu\left(\left\{x \in \mathbb{R}^{n} ;|\varphi(x)| \geq C_{2} \sqrt{n}(\log n)^{2+\varepsilon / 2}\|\varphi\|_{L_{1}(\mu)}\right\}\right)=0 .
$$

From (103) and (105) we conclude that, for any $t \geq 1$, we have

$$
\mu\left(\left\{x \in \mathbb{R}^{n} ;|\varphi(x)|>t\|\varphi\|_{L_{1}(\mu)}\right\}\right) \leq e^{-C^{\prime} \frac{t^{2}}{\log ^{4} \varepsilon \varepsilon(t+1)}}
$$

for an appropriate constant $C^{\prime}$ depending only on $\alpha$ and $\varepsilon$. Inequality (106) is precisely the desired estimate (102). Thus, the lemma is proved, for the case where $n \geq \max \left\{\frac{2}{c_{1}}, 4\right\}$.

To deal with the case where $1 \leq n \leq \max \left\{\frac{2}{c_{1}}, 4\right\}$, simply note that $\|\varphi\|_{L_{\infty}(\mu)} \leq$ $\tilde{C} n\|\varphi\|_{L_{1}(\mu)}$, and hence (102) is trivially true in this degenerate case.

Theorem 1.3 is the case of $\varepsilon=1$ in the following theorem.

Theorem 4.6. Let $\alpha>0,0<\varepsilon \leq 1$, let $n \geq 1$ be an integer, and let $f: \mathbb{R}^{n} \rightarrow[0, \infty)$ be an $\alpha$-concave function with $\int f=1$. Denote by $\mu$ the probability measure whose density is $f$. Then there exists a nonzero linear functional $\varphi: \mathbb{R}^{n} \rightarrow \mathbb{R}$ such that, for any $t \geq 1$, we have

$$
\mu\left(\left\{x \in \mathbb{R}^{n} ;|\varphi(x)|>t\|\varphi\|_{L_{1}(\mu)}\right\}\right)<e^{-c_{\alpha, \varepsilon} \frac{t^{2}}{\log ^{4}+\varepsilon(t+1)}},
$$

where $c_{\alpha, \varepsilon}>0$ is a constant that depends only on $\alpha$ and $\varepsilon$.

Proof. In this proof, $c, c_{1}, C, c^{\prime}$, etc. denote positive constants that depend only on $\alpha$ and $\varepsilon$. Putting $f^{\prime}(x)=f(-x)$, we consider the convolution $g=f * f^{\prime}$. The function $g: \mathbb{R}^{n} \rightarrow[0, \infty)$ is an even, $(2 \alpha+1) n$-concave function.

Denote by $\nu$ the measure whose density is $g$. By Lemma 4.5, there exists a nonzero linear functional $\varphi: \mathbb{R}^{n} \rightarrow \mathbb{R}$ such that

$$
\nu\left(\left\{x \in \mathbb{R}^{n} ;|\varphi(x)|>t\|\varphi\|_{L_{1}(\nu)}\right\}\right)<e^{-c \frac{t^{2}}{\log ^{4}+\varepsilon}(t+1)}
$$

for any $t \geq 1$. Let $M>0$ be such that $\mu\left(\left\{x \in \mathbb{R}^{n} ;|\varphi(x)| \leq M\right\}\right)=\frac{2}{3}$. Arguing as in the proof of Theorem 4.3, we find that $M \geq c\|\varphi\|_{L_{1}(\nu)}$. Let $X$ and $Y$ be independent random vectors distributed according to the densities $f$ and $f^{\prime}$, respectively. Then the random vector $X+Y$ is distributed according to the density $g$. As in the proof of Theorem 4.3, we conclude that, for any $t \geq 1$,

$$
\begin{aligned}
& \frac{2}{3} \operatorname{Prob}\{|\varphi(X)| \geq(t+1) M\} \\
& \quad \leq \operatorname{Prob}\left\{|\varphi(X+Y)| \geq c t\|\varphi\|_{L_{1}(\nu)}\right\} \leq e^{-\tilde{c} \frac{t^{2}}{\log ^{4} \varepsilon(t+1)}} .
\end{aligned}
$$

By adjusting the constants, we conclude that

$$
\mu\left(\left\{x \in \mathbb{R}^{n} ;|\varphi(x)|>t\|\varphi\|_{L_{1}(\mu)}\right\}\right)<e^{-\hat{c} \frac{t^{2}}{\log ^{4}+\varepsilon(t+1)}}
$$

for any $t \geq 1$. The theorem is proved. 
Theorem 1.1 is the case of $\varepsilon=1$ in the following theorem.

Theorem 4.7. Let $0<\varepsilon \leq 1$, let $n \geq 1$ be an integer, and let $K \subset \mathbb{R}^{n}$ be a convex body of volume one. Then there exists a nonzero linear functional $\varphi: \mathbb{R}^{n} \rightarrow \mathbb{R}$ such that

$$
\operatorname{Vol}_{n}\left(\left\{x \in K ;|\varphi(x)|>t\|\varphi\|_{L_{1}(K)}\right\}\right)<e^{-c_{\varepsilon} \frac{t^{2}}{\log ^{4+\varepsilon}(t+1)}}
$$

for any $t \geq 1$. Equivalently, denote $\psi_{2^{-}}(t)=\exp \left(\frac{t^{2}}{\log ^{4+\varepsilon}(t+5)}\right)-1$. Then the linear functional $\varphi$ satisfies

$$
\|\varphi\|_{L_{\psi_{2}}(K)} \leq C_{\varepsilon}\|\varphi\|_{L_{1}(K)} .
$$

Here, $C_{\varepsilon}, c_{\varepsilon}>0$ are constants that depend only on $\varepsilon$.

Proof. The function $1_{K}$ is $n$-concave. Thus, by Theorem 4.6, there exists a nonzero linear functional $\varphi: \mathbb{R}^{n} \rightarrow \mathbb{R}^{n}$ that satisfies (108). To establish the second part, note that, for $M=\|\varphi\|_{L_{1}(K)}$,

$$
\begin{aligned}
\int_{K} \exp & {\left[\left(\frac{\varphi(x)}{C_{1} M}\right)^{2} / \log ^{4+\varepsilon}\left(\frac{|\varphi(x)|}{C_{1} M}+5\right)\right] d x } \\
& =\int_{0}^{\infty} \operatorname{Vol}_{n}\left(\left\{x \in K ;\left(\frac{\varphi(x)}{C_{1} M}\right)^{2} / \log ^{4+\varepsilon}\left(\frac{|\varphi(x)|}{C_{1} M}+5\right)>\log t\right\}\right) d t \\
& \leq \int_{0}^{\infty} \min \left\{1, \frac{1}{t^{c C_{1}}}\right\} d t \leq 2
\end{aligned}
$$

for a suitable constant $C_{1}>0$.

\section{$\S 5$. USING $\ell$-POSITION}

In this section we employ the fundamental properties of the $\ell$-position; see, e.g., [31, $\S \S 2$ and 3]. We shall prove variants of Theorem 1.1 and Theorem 1.3, where rather than asserting the existence of a linear functional with a certain property, we shall show that a "random" linear functional possesses this property. See Theorem 5.1 and Theorem 5.2 below for the exact formulation.

We denote by $\sigma_{n}$ the unique rotation invariant probability measure on the unit sphere $S^{n-1}$. Let $K \subset \mathbb{R}^{n}$ be a centrally symmetric convex body. For $x \in \mathbb{R}^{n}$ we put $\|x\|_{K}=$ $\inf \{\lambda>0 ; x \in \lambda K\}$. Then $\|\cdot\|_{K}$ is the norm whose unit ball is $K$. We define

$$
M(K)=\int_{S^{n-1}}\|x\|_{K} d \sigma_{n}(x) .
$$

A well-known consequence of Hölder's inequality is that $M(K) \mathrm{v} \cdot \operatorname{rad} .(K) \geq 1$. In general, $M(K)$ may be much larger than $1 / \mathrm{v} \cdot \operatorname{rad} .(K)$. However, a fundamental theorem, which follows from the results of Lewis, Figiel and Tomczak-Jaegermann and Pisier (see, e.g., $[27, \S \S 14$ and 15$]$ or $[31, \S \S 2$ and 3$])$ states that for any centrally symmetric convex body $K \subset \mathbb{R}^{n}$, there exists a linear transformation $u \in S L_{n}(\mathbb{R})$ such that

$$
M(u(K)) \leq C \frac{\log n}{\operatorname{v.rad}(u(K))} .
$$

It is customary to say that this linear map $u$ transforms $K$ into $\ell$-position, or that the body $u(K)$ is in $\ell$-position.

Theorem 5.1. Let $0<\varepsilon \leq 1$, let $n \geq 1$, and let $f: \mathbb{R}^{n} \rightarrow[0, \infty)$ be a log-concave function with $\int f=1$ such that $\int x f(x) d x=0$. Then there exists $u \in S L_{n}(\mathbb{R})$ for which the following is true. 
Denote $\tilde{f}=f \circ u$, and let $\mu$ be the measure whose density is $\tilde{f}$. Then, there exists $\Theta \subset S^{n-1}$ with $\sigma_{n}(\Theta) \geq \frac{4}{5}$ such that, for any $\theta \in \Theta$ and any $1 \leq t \leq \sqrt{n} \log ^{3+\varepsilon / 2} n$, we have

$$
\mu\left(\left\{x \in \mathbb{R}^{n} ;|\langle x, \theta\rangle|>t\|\langle\cdot, \theta\rangle\|_{L_{1}(\mu)}\right\}\right)<e^{-c_{\varepsilon} \frac{t^{2}}{\log ^{2}(n+1) \log 4+\varepsilon(t+1)}},
$$

where $c_{\varepsilon}>0$ is a constant depending only on $\varepsilon$.

Proof. In this proof, the letters $c, C, \tilde{C}$, etc. stand for constants depending solely on $\varepsilon$. We define $f^{\prime}(x)=f(-x)$ and $S(f)(x)=\left(f * f^{\prime}\right)(x)=\int_{\mathbb{R}^{n}} f(y) f(y-x) d y$. Then $S(f)$ is an even, log-concave function with $\int S(f)=1$. Clearly, for any map $u \in S L_{n}(\mathbb{R})$ we have $S(f) \circ u=S(f \circ u)$. Recall the definition (46) of $\hat{T}_{\varepsilon}$. Then

$$
\hat{T}_{\varepsilon}(S(f \circ u))=u^{*}\left(\hat{T}_{\varepsilon}(S(f))\right) .
$$

The set $\hat{T}_{\varepsilon}(S(f))$ is convex and centrally symmetric. Let $u \in S L_{n}(\mathbb{R})$ be such that $u^{*}\left(\hat{T}_{\varepsilon}(S(f))\right)$ is in $\ell$-position. We write $\tilde{f}=f \circ u$.

To simplify the notation, we denote $g=S(\tilde{f})$. By Lemma 4.2,

$$
\text { v. } \operatorname{rad} .\left(\hat{T}_{\varepsilon}(g)\right) \geq c \frac{g(0)^{1 / n}}{L_{g}} .
$$

By our assumption, $\hat{T}_{\varepsilon}(g)$ is in $\ell$-position. Therefore,

$$
M\left(\hat{T}_{\varepsilon}(g)\right) \leq C \frac{L_{g} \log n}{g(0)^{1 / n}} .
$$

Inequality (110) implies the existence of a set $\Theta_{1} \subset S^{n-1}$ with $\sigma_{n}\left(\Theta_{1}\right) \geq \frac{9}{10}$ such that

$$
c \frac{g(0)^{1 / n}}{L_{g} \log n} \theta \in \hat{T}_{\varepsilon}(g)
$$

for each $\theta \in \Theta_{1}$. Denote $T=\left\{x \in \mathbb{R}^{n} ; \Upsilon g(x) \leq \log 2\right\}$. Arguing as in (90) and (91), we see that

$$
\text { v. } \operatorname{rad} .(T) \leq c \frac{g(0)^{1 / n}}{L_{g}} .
$$

The upper bound on v.rad.(T) implies the existence of $\Theta_{2} \subset S^{n-1}$ with $\sigma_{n}\left(\Theta_{2}\right) \geq \frac{9}{10}$ such that for each $\theta \in \Theta_{2}$,

$$
C \frac{g(0)^{1 / n}}{L_{g}} \theta \notin T
$$

(otherwise, the convex hull of $T \cap C \frac{g(0)^{1 / n}}{L_{g}} S^{n-1}$ and the origin, which is contained in $T$, would have too large a volume). We set $\Theta=\Theta_{1} \cap \Theta_{2}$. Then $\sigma_{n}(\Theta) \geq \frac{4}{5}$. We compare (111) and (112), and conclude that for any $\theta \in \Theta$ we have

$$
\forall s \in \mathbb{R}, \quad s \theta \in T \Rightarrow \frac{c^{\prime} s}{\log n} \theta \in \hat{T}_{\varepsilon}(g) .
$$

All that remains for us to show is that

$$
\mu\left(\left\{x \in \mathbb{R}^{n} ;|\langle x, \theta\rangle|>t\|\langle\cdot, \theta\rangle\|_{L_{1}(\mu)}\right\}\right)<e^{-c \frac{t^{2}}{\log ^{2}(n+1) \log ^{4}+2 \varepsilon(t+1)}}
$$

for any $\theta \in \Theta$, and any $1 \leq t \leq \sqrt{n} \log ^{3+\varepsilon} n$. We thus focus our attention on establishing (114). Fix $\theta \in \Theta$ and set

$$
M=\|\langle\cdot, \theta\rangle\|_{L_{1}(\mu)}=\int_{\mathbb{R}^{n}}|\langle x, \theta\rangle| \tilde{f}(x) d x .
$$


Let $\nu$ denote the measure whose density is $g$. Arguing as in (99) above, we find that

$$
M \geq C\|\langle\cdot, \theta\rangle\|_{L_{1}(\nu)} .
$$

Borell's lemma (17) implies now that $\frac{\theta}{C M} \in T$, and by (113) we deduce that

$$
\frac{\theta}{C^{\prime} M \log n} \in \hat{T}_{\varepsilon}(g) \text {. }
$$

We may apply Lemma 3.2, based on (115). We conclude that

$$
\nu\left(\left\{y \in \mathbb{R}^{n} ;|\langle x, y\rangle|>C M(\log n) s\right\}\right) \leq e^{-C^{\prime} \frac{s^{2}}{\log ^{4}+\varepsilon(s+1)}}
$$

for any $1 \leq s \leq \sqrt{n} \log ^{2+\varepsilon / 2} n$. Estimate (116) is very close to our desired inequality (114), the only difference being that the measure $\mu$ is replaced by $\nu$. Next, we repeat the argument (100) from the proof of Theorem 4.3, and deduce (114) from (116).

Let $X$ and $Y$ be independent random vectors that are distributed according to the densities $\tilde{f}$ and $\tilde{f}^{\prime}(x)=\tilde{f}(-x)$, respectively. Then the random vector $X+Y$ is distributed according to the density $g$. Note that $\operatorname{Prob}\{|\langle Y, \theta\rangle| \leq 3 M\} \geq \frac{2}{3}$. Consequently, for any $C \log ^{2} n \leq s \leq \sqrt{n} \log ^{3+\varepsilon} n$, we have

$$
\begin{aligned}
\operatorname{Prob} & \{|\langle X, \theta\rangle| \geq(s+1) M\} \\
& \leq \frac{3}{2} \operatorname{Prob}\{|\langle X+Y, \theta\rangle| \geq s M\} \leq e^{-C^{\prime} \frac{s^{2}}{\log ^{2}(n+1) \log ^{4+2 \varepsilon}(s+1)}} .
\end{aligned}
$$

Now (114) follows easily, in the range $C \log ^{2} n \leq t \leq c \sqrt{n} \log ^{3+\varepsilon} n$. Note that (114) in the range $1 \leq t \leq C \log ^{2} n$ is redundant, and (17) provides a better estimate in that range. The proof is complete.

Theorem 5.2. Let $0<\varepsilon \leq 1, \alpha>0$, let $n \geq 1$ be an integer, and let $f: \mathbb{R}^{n} \rightarrow[0, \infty)$ be an $\alpha$-concave function with $\int f=1$ such that $\int x f(x) d x=0$. Then there exists $u \in S L_{n}(\mathbb{R})$ for which the following is true.

Denote $\tilde{f}=f \circ u$, and let $\mu$ be the measure whose density is $\tilde{f}$. Then there exists $\Theta \subset S^{n-1}$ with $\sigma_{n}(\Theta) \geq \frac{4}{5}$ such that, for any $\theta \in \Theta$ and any $t \geq 1$, we have

$$
\mu\left(\left\{x \in \mathbb{R}^{n} ;|\langle x, \theta\rangle|>t\|\langle\cdot, \theta\rangle\|_{L_{1}(\mu)}\right\}\right)<e^{-c_{\varepsilon, \alpha} \frac{t^{2}}{\log ^{2}(n+1) \log ^{4+\varepsilon}(t+1)}},
$$

where $c_{\varepsilon, \alpha}>0$ is a constant depending only on $\varepsilon$ and $\alpha$.

Proof. The argument is similar to the proof of Lemma 4.5. We outline the details. In this proof we denote by $c, C, c^{\prime}, \hat{c}$, etc. constants depending only on $\varepsilon$ and $\alpha$. An $\alpha n$-concave function is also log-concave. We thus may employ Theorem 5.1. Let $u \in S L_{n}(\mathbb{R})$ be the linear map from the conclusion of that theorem. We put $\tilde{f}=f \circ u$, and note that the barycenter of $\tilde{f}$ lies at the origin.

By the conclusion of Theorem 5.1, there exists $\Theta \subset S^{n-1}$ with $\sigma_{n}(\Theta) \geq \frac{4}{5}$ such that

$$
\mu\left(\left\{x \in \mathbb{R}^{n} ;|\langle x, \theta\rangle|>t\|\langle\cdot, \theta\rangle\|_{L_{1}(\mu)}\right\}\right)<e^{-c \frac{t^{2}}{\log ^{2}(n+1) \log ^{4+\varepsilon}(t+1)}}
$$

for any $\theta \in \Theta$ and any $1 \leq t \leq \sqrt{n} \log ^{3+\varepsilon / 2} n$. We substitute $t=\sqrt{n} \log ^{3+\varepsilon / 2} n$ in (117) and then use Lemma 4.4 (as in (104) and (105)). By the conclusion of that lemma, for any $\theta \in \Theta$, we have

$$
\mu\left(\left\{x \in \mathbb{R}^{n} ;|\langle x, \theta\rangle| \geq C_{2} \sqrt{n}(\log n)^{3+\varepsilon / 2}\|\langle\cdot, \theta\rangle\|_{L_{1}(\mu)}\right\}\right)=0,
$$

under the assumption that $n \geq C$. The theorem follows from (117) and (118).

Recall that the characteristic function of a convex set is trivially $n$-concave. The following corollary is equivalent to the case of $\varepsilon=\alpha=1$ in Theorem 5.2. 
Corollary 5.3. Let $n \geq 1$ be an integer, and let $K \subset \mathbb{R}^{n}$ be a convex body of volume one whose barycenter lies at the origin. Then there exists $u \in S L_{n}(\mathbb{R})$ for which the following is true.

Denote $\tilde{K}=u(K)$. Then, there exists $\Theta \subset S^{n-1}$ with $\sigma_{n}(\Theta) \geq \frac{4}{5}$ such that, for any $\theta \in \Theta$ and any $t \geq 1$,

$$
\operatorname{Vol}_{n}\left(\left\{x \in \tilde{K} ;|\langle x, \theta\rangle|>t\|\langle\cdot, \theta\rangle\|_{L_{1}(\tilde{K})}\right\}\right)<e^{-c \frac{t^{2}}{\log ^{2}(n+1) \log ^{5}(t+1)}},
$$

where $c>0$ is a universal constant.

Added in proofs. Very recently, Giannopoulos, Pajor and Paouris have simplified and slightly improved our proof. They observed that it is possible to obtain an estimate as in Lemma 3.6 above in a direct way, without appealing to Milman's ellipsoid. This leads to better powers of the logarithms in our main results. See http://arxiv.org/abs/math.FA/ 0604299.

\section{REFERENCES}

[1] K. Ball, Isometric problems in $\ell_{p}$ and sections of convex sets, Ph.D. Dissertation, Trinity College, Cambridge, 1986.

[2] F. Barthe, O. Guédon, S. Mendelson, and A. Naor, A probabilistic approach to the geometry of the $l_{p}^{n}$-ball, Ann. Probab. 33 (2005), 480-513. MR2123199 (2006g:46014)

[3] S. G. Bobkov, On concentration of distributions of random weighted sums, Ann. Probab. 31 (2003), no. 1, 195-215. MR1959791 (2004a:60049)

[4] S. G. Bobkov and F. L. Nazarov, On convex bodies and log-concave probability measures with unconditional basis, Geometric Aspects of Functional Analysis, Lecture Notes in Math., vol. 1807, Springer, Berlin, 2003, pp. 53-69. MR2083388 (2005k:60058)

[5] — Large deviations of typical linear functionals on a convex body with unconditional basis, Stochastic Inequalities and Applications, Progr. Probab., vol. 56, Birkhäuser, Basel, 2003, pp. 3-13. MR2073422 (2005f:52013)

[6] C. Borell, Convex measures on locally convex spaces, Ark. Mat. 12 (1974), 239-252. MR0388475 $(52: 9311)$

[7] _ Convex set functions in d-space, Period. Math. Hungar. 6 (1975), no. 2, 111-136. MR0404559 (53:8359)

[8] J. Bourgain, On the distribution of polynomials on high-dimensional convex sets, Geometric Aspects of Functional Analysis (1989-90), Lecture Notes in Math., vol. 1469, Springer, Berlin, 1991, pp. 127137. MR1122617 (92j:52007)

[9] _ On the isotropy-constant problem for "PSI-2"-bodies, Geometric Aspects of Functional Analysis, Lecture Notes in Math., vol. 1807, Springer, Berlin, 2003, pp. 114-121. MR2083391 (2006c:46011)

[10] J. Bourgain and V. Milman, New volume ratio properties for convex symmetric bodies in $R^{n}$, Invent. Math. 88 (1987), no. 2, 319-340. MR0880954 (88f:52013)

[11] H. Brascamp and E. Lieb, On extensions of the Brunn-Minkowski and Prékopa-Leindler theorems, including inequalities for log concave functions, and with an application to the diffusion equation, J. Funct. Anal. 22 (1976), no. 4, 366-389. MR0450480 (56:8774)

[12] Yu. S. Davidovich, B. I. Korenblyum, and B. I. Khatset, A certain property of logarithmically concave functions, Dokl. Akad. Nauk SSSR 185 (1969), no. 6, 1215-1218; English transl., Soviet Math. Dokl. 10 (1969), 477-480. MR0241584 (39:2924)

[13] A. Dinghas, Über eine Klasse superadditiver Mengenfunktionale von Brunn-Minkowski-Lusternikschem Typus, Math. Z. 68 (1957), 111-125. MR0096173 (20:2668)

[14] _ Über zwei allgemeine Sätze von Brunn-Minkowski-Lusternikschem Typus, Norske Vid. Selsk. Forh., Trondheim 28 (1955), 182-185 (1956). MR0079788 (18:146e)

[15] B. Grünbaum, Partitions of mass-distributions and of convex bodies by hyperplanes, Pacific J. Math. 10 (1960), 1257-1261. MR0124818 (23:A2128)

[16] R. Henstock and A. M. Macbeath, On the measure of sum-sets. I. The theorems of Brunn, Minkowski, and Lusternik, Proc. London Math. Soc. (3) 3 (1953), 182-194. MR0056669 (15:109g)

[17] B. Klartag, A geometric inequality and a low M-estimate, Proc. Amer. Math. Soc. 132 (2004), no. 9, 2619-2628. MR2054787 (2004m:46029)

[18] _ An isomorphic version of the slicing problem, J. Funct. Anal. 218 (2005), 372-394. MR2108116 (2005i:52007) 
[19] _ Marginals of geometric inequalities, Geometric Aspects of Functional Analysis, Israel Seminar, Lecture Notes in Math., Springer (to appear).

[20] - On convex perturbations with a bounded isotropic constant, Geom. Funct. Anal. 16 (2006), no. 6, 1274-1290. MR2276540 (2007i:52005)

[21] B. Klartag and V. Milman, Geometry of log-concave functions and measures, Geom. Dedicata 112 (2005), 169-182. MR2163897 (2006d:52004)

[22] L. Leindler, On a certain converse of Hölder's inequality, Linear Operators and Approximation (Proc. Conf., Oberwolfach, 1971), Internat. Ser. Numer. Math., vol. 20, Birkhäuser, Basel, 1972, pp. 182-184. MR0430188 (55:3195)

[23] C. G. Lekkerkerker, A property of logarithmic concave functions. I, II, Indag. Math. 15 (Nederl. Akad. Wetensch. Proc. Ser. A. 56) (1953), 505-521. MR0059977 (15:608d)

[24] M. Meyer and A. Pajor, On Santaló's inequality, Geometric Aspects of Functional Analysis (198788), Lecture Notes in Math., vol. 1376, Springer, Berlin, 1989, pp. 261-263. MR1008727 (90h:52012)

[25] V. Milman, Randomness and pattern in convex geometric analysis, Proceedings of the International Congress of Mathematicians, Vol. II (Berlin, 1998), Doc. Math. 1998, Extra Vol. II, 665-677. MR1648115 (2000k:52001)

[26] V. Milman and A. Pajor, Isotropic position and inertia ellipsoids and zonoids of the unit ball of a normed n-dimensional space, Geometric Aspects of Functional Analysis, Israel Seminar (1987-88), Lecture Notes in Math., vol. 1376, Springer, Berlin, 1989, pp. 64-104. MR1008717 (90g:52003)

[27] V. Milman and G. Schechtman, Asymptotic theory of finite-dimensional normed spaces, Lecture Notes in Math., vol. 1200, Springer-Verlag, Berlin, 1986. MR0856576 (87m:46038)

[28] G. Paouris, $\Psi_{2}$-estimates for linear functionals on zonoids, Geometric Aspects of Functional Analysis, Lecture Notes in Math., vol. 1807, Springer, Berlin, 2003, pp. 211-222. MR2083399 (2005g:52021)

[29] (2005), no. 3, 285-299. MR2146128 (2006c:52004)

[30] Concentration of mass on isotropic convex bodies, C. R. Math. Acad. Sci. Paris 342 (2006), no. 3, 179-182. MR2198189 (2006h:52001)

[31] G. Pisier, The volume of convex bodies and Banach space geometry, Cambridge Tracts in Math., vol. 94, Cambridge Univ. Press, Cambridge, 1989. MR1036275 (91d:52005)

[32] A. Prékopa, Logarithmic concave measures with application to stochastic programming, Acta Sci. Math. (Szeged) 32 (1971), 301-316. MR0315079 (47:3628)

[33] _ On logarithmic concave measures and functions, Acta Sci. Math. (Szeged) 34 (1973), 335343. MR0404557 (53:8357)

[34] L. A. Santaló, An affine invariant for convex bodies of $n$-dimensional space, Portugaliae Math. 8 (1949), 155-161. (Spanish) MR0039293 (12:526f)

[35] R. Schneider, Convex bodies: the Brunn-Minkowski theory, Encyclopedia Math. Appl., vol. 44, Cambridge Univ. Press, Cambridge, 1993. MR1216521 (94d:52007)

School of Mathematics, Institute for Advanced Study, Einstein Drive, Princeton, New JERSEY 08540

E-mail address: bklartag@math.princeton.edu

Received 1/AUG/2006

Originally published in English 